\title{
A priori DNS study of applicability of flamelet concept to predicting mean concentrations of species in turbulent premixed flames at various Karlovitz numbers
}

\author{
A.N. Lipatnikov ${ }^{1}$, V.A. Sabelnikov²,3, F.E. Hernández-Pérez ${ }^{4}$, W. Song ${ }^{4}$, Hong G. $\mathrm{Im}^{4}$ \\ ${ }^{1}$ Department of Mechanics and Maritime Sciences, Chalmers University of Technology, Gothenburg, 41296 Sweden \\ ${ }^{2}$ ONERA - The French Aerospace Laboratory, F-91761 Palaiseau, France \\ ${ }^{3}$ Central Aerohydrodynamic Institute (TsAGI), 140180 Zhukovsky, Moscow Region, Russian Federation \\ ${ }^{4}$ Clean Combustion Research Center, King Abdullah University of Science and Technology, \\ Thuwal 23955-6900, Saudi Arabia
}

\begin{abstract}
Complex-chemistry direct numerical simulation (DNS) data obtained earlier from lean hydrogen-air flames associated with corrugated flame (case A), thin reaction zone (case B), and broken reaction zone (case C) regimes of turbulent burning are analysed to directly assess capabilities of the flamelet approach to predict mean concentrations of species in a premixed turbulent flame. The approach consists in averaging dependencies of mole fractions, reaction rates, temperature, and density on a single combustion progress variable $c$, which are all obtained from the unperturbed laminar flame. For this purpose, four alternative definitions of $c$ are probed and two probability density functions (PDFs) are adopted, i.e. either an actual PDF extracted directly from the DNS data or a presumed $\beta$-function PDF obtained using the DNS data on the first two moments of the $c(\mathbf{x}, t)$-field. Results show that the mean density and mean mole fractions of $\mathrm{H}_{2}, \mathrm{O}_{2}$, and $\mathrm{H}_{2} \mathrm{O}$ are well predicted using both PDFs for each $c$, although the predictive capabilities are little worse in case C. In cases A and B, the use of the actual PDF and the fuel-based $c$ also offers an opportunity to well predict mean mole fractions of $\mathrm{O}$ and $\mathrm{H}$, whereas the mean mole fraction of $\mathrm{OH}$ is slightly underestimated. In the highly turbulent case $\mathrm{C}$, the same approach performs worse, but still appears to be acceptable for evaluating the mean radical concentrations. The use of the $\beta$-function PDFs or another combustion progress variable yields substantially worse results for these radicals. When compared to the mean mole fractions, the mean rate of product creation, i.e. the source term in the transport equation for the mean combustion progress variable, is worse predicted even for a quantity (species concentration or temperature) adopted to define $c$ and using the actual PDF. Consequently, turbulent burning velocity is not predicted either.
\end{abstract}

Keywords: premixed turbulent combustion, complex chemistry, modeling, DNS

\section{Introduction}

Due to strict legislation on emissions from gas turbine and piston engines, computational prediction of concentrations of various species (not only reactants and major products, but also intermediate species such as $\mathrm{CO}$, radicals $\mathrm{O}, \mathrm{H}, \mathrm{OH}$, etc.) in a premixed or stratified turbulent flame has been developed and employed in laboratory and device scale simulations. Many such turbulent combustion models adopt the flamelet concept, as summarized, e.g., in Table 4 in Ref. [1] or Tables 5 and 6 in Ref. [2]. The concept utilizes results (the so-called flamelet library) of numerical simulations of a set of laminar premixed flames, performed by invoking appropriately detailed models of molecular transport and chemical kinetics. Such results could be filtered and directly adopted in large eddy simulation (LES) within the framework of filtered tabulated chemistry for LES, e.g. see F-TACLES model [3]. Alternatively, by using flame prolongation of intrinsic low-dimensional manifold (FPI) [4] or 
flamelet generated manifold (FGM) [5] techniques, a flamelet library is often stored in a form of dependencies of temperature $T_{L}(\xi)$, density $\rho_{L}(\xi)$, mass $Y_{n, L}(\xi)$ or mole $X_{n, L}(\xi)$ fractions, and mass rates $M_{n} W_{n, L}(\xi)$ of consumption/production of $n=$ $1, \ldots, N$ species on a set $\xi$ of independent variables. Here, $M_{n}$ is the molecular weight of species $n$, the rate $W_{n, L}$ is expressed in mole/ $\left(\mathrm{cm}^{3} \mathrm{~s}\right)$, and the set $\xi$ may consist of a single combustion progress variable $c$, which varies from zero in fresh reactants to unity in equilibrium combustion products, but may also involve mixture fraction in the case of stratified combustion, mixture enthalpy in the case of non-adiabatic flames, pressure in the case of non-isobaric burning (e.g. in a piston engine), stretch rate in highly turbulent flames, etc. In the rest of the present paper, the simplest case of a single independent scalar variable $c$ will be considered.

To adopt a flamelet library in Reynolds-averaged Navier-Stokes (RANS) or LES studies of turbulent flames, the library should be coupled with a model that incorporates the influence of turbulence on combustion. Such a coupling is commonly implemented using the following statistical averaging in RANS application:

$$
\begin{gathered}
\bar{W}_{n}(\mathbf{x}, t)=\int_{0}^{1} W_{n, L}(c) P(c, \mathbf{x}, t) d c, \\
\bar{X}_{n}(\mathbf{x}, t)=\int_{0}^{1} X_{n, L}(c) P(c, \mathbf{x}, t) d c
\end{gathered}
$$

or a similar form of filtering operation in LES applications to determine the mean or filtered production/consumption rates and mole fractions, respectively. For brevity, only the RANS formulation will be discussed in this study although the results can be extended to the LES framework. To complete the model, the probability density function (PDF) $P(c, \mathbf{x}, t)$ for the combustion progress variable is found by solving an appropriately closed transport equation [6-9] for $P(c, \mathbf{x}, t)$ or by invoking a presumed PDF, where the latter is most commonly used in large scale simulations of premixed or stratified turbulent combustion in engines in favor of its computational efficiency.

In general, the presumed PDF approach [10-13] consists of (i) assuming a general shape of the PDF, which involves a few unknown parameters, and (ii) evaluating these parameters by comparing values of the first moments of the $c(\mathbf{x}, t)$-field, calculated using the PDF, with the values of these moments, obtained by solving appropriate transport equations, e.g. for the Reynolds-averaged $\bar{c}(\mathbf{x}, t)$ or the Favre-averaged $\tilde{c}(\mathbf{x}, t) \equiv \overline{\rho c}(\mathbf{x}, t) / \bar{\rho}(\mathbf{x}, t)$ and $\overline{c^{2}}(\mathbf{x}, t)$ or $\widetilde{c^{2}}(\mathbf{x}, t) \equiv \overline{\rho c^{2}}(\mathbf{x}, t) / \bar{\rho}(\mathbf{x}, t)$, respectively. Specifically, (i) the mean source terms $\bar{W}_{c}(\mathbf{x}, t)$ and $\overline{c W}_{c}(\mathbf{x}, t)$ in the aforementioned transport equations are closed invoking the presumed PDF, (ii) the transport equations are numerically integrated, (iii) the PDF parameters are recalculated using the obtained moments of the $c(\mathbf{x}, t)$-field, and, finally, (iv) Eq. (2) is applied to evaluate mean concentrations of various species.

The PDF shape may be expressed as a sum of Dirac delta functions [10], as various combinations of Dirac delta functions and a flamelet PDF [11,12], or, most commonly, as the following $\beta$-function $[11,13]$

$$
\begin{aligned}
& P_{\beta}\left(c, \bar{c}, \overline{c^{2}}\right)=\frac{\Gamma(a+b)}{\Gamma(a) \Gamma(b)} c^{a-1}(1-c)^{b-1}, \\
& a=\bar{c}\left(\frac{1}{g}-1\right), \quad b=(1-\bar{c})\left(\frac{1}{g}-1\right) .
\end{aligned}
$$


Here, $g=\overline{c^{\prime 2}} /[\bar{c}(1-\bar{c})]$ is the segregation factor, $\overline{c^{\prime 2}}=\overline{c^{2}}-\bar{c}^{2}$, and the gamma function $\Gamma(a)=\int_{0}^{\infty} \zeta^{a-1} \mathrm{e}^{-\zeta} d \zeta$ is required to satisfy the normalization constraint of $\int_{0}^{1} P(c) d c=1$. Henceforth, dependencies of $\bar{c}, \overline{c^{2}}, g, a, b$, etc. on $\mathbf{x}$ and $t$ are not specified for brevity. To model the mass-weighted $\operatorname{PDF} \tilde{P}(c) \equiv \rho(c) P(c) / \bar{\rho}$, the $\operatorname{PDF} P_{\beta}\left(c, \bar{c}, \overline{c^{2}}\right)$ and quantities $\bar{c}$ and $g$ in Eqs. (3) and (4) are substituted with $\tilde{P}_{\beta}\left(c, \tilde{c}, \widetilde{c^{2}}\right), \tilde{c}$, and $\tilde{g}=\left(\widetilde{c^{2}}-\tilde{c}^{2}\right) /[\tilde{c}(1-\tilde{c})]$, respectively. The latter option is often preferred in reacting flows, because transport equations for $\tilde{c}$ and $\widetilde{c^{2}}$ involve a fewer number of unclosed terms than those for $\bar{c}$ and $\overline{c^{2}}$. From the fundamental perspective, the use of direct statistics or mass-weighted statistics in Eqs. (1) and (2) is equally justified. Accordingly, both $P_{\beta}\left(c, \bar{c}, \overline{c^{2}}\right)$ and $\widetilde{P}_{\beta}\left(c, \tilde{c}, \widetilde{c^{2}}\right)$ are addressed in the present work. In applied CFD research, the presumed $\beta$-function PDF is widely adopted because its shape is flexible enough, depending on the values of $a$ and $b$, to vary from a quasi-bi-modal PDF $(g \rightarrow 1)$ representing the flamelet regime of premixed turbulent combustion [14] to a quasiGaussian PDF $(g \ll 1)$ representing highly turbulent conditions. Moreover, the numerical efficiency of the approach benefits from the simple algebraic relations given by Eq. (4).

In spite of the wide use of the flamelet concept coupled with a presumed PDF [10-13,15-32], such an approach requires further study. In particular, its validation has been mainly performed in a posteriori RANS [15,17,22] or LES studies [16,18$21,23,24,27,28,30-32]$, with the reported results showing limited capabilities of the approach for predicting mean concentrations of intermediate species such as $\mathrm{H}_{2}$ (in hydrocarbon-air flames), $\mathrm{CO}, \mathrm{OH}$, or $\mathrm{CH}_{2} \mathrm{O}$ (see Fig. 18 in Ref. [17], Fig. 24 in Ref. [21], Fig. 10 in Ref. [22], Fig. 25 in Ref. [23], Fig. 9 in Ref. [24], Figs. 11 and 12 in Ref. [27], Figs. 20 and 21 in Ref. [28], Fig. 18 in Ref. [30], or Fig. 5 in Ref. [31]). However, these results are not sufficient to draw an overall negative conclusion regarding the flamelet concept. For instance, predictive capabilities of Eqs. (1)-(3) can be significantly different [33]. Moreover, substantial disagreement between RANS/LES simulated and measured or direct numerical simulation (DNS) data, observed in the aforementioned figures, could stem not only from eventual limitations of the flamelet concept and/or the invoked PDF, but also from limitations of other models adopted in a posteriori study. For instance, as reviewed elsewhere $[34,35]$, capabilities of available models for predicting effects of combustion-induced thermal expansion on turbulence in premixed flames are limited and could account for the disagreement discussed here. Moreover, when comparing experimental and numerical data, the disagreement could stem from uncertainties due to boundary and initial conditions, reaction mechanisms, heat losses, etc.

In consideration of the above, there is need for further a priori investigation and assessment of the predictive capabilities of Eq. (1), Eq. (2), and/or Eqs. (3) and (4) in a target-directed and comprehensive manner under various conditions. Such an assessment appears to be of particular interest, because recent experimental and DNS data reviewed by Driscoll et al. [36] suggest that the domain of the flamelet concept validity is substantially wider than it was assumed earlier. This hypothesis results from comparison of profiles of conditioned quantities extracted from highly turbulent flames with the counterpart profiles obtained from laminar flames. The hypothesis implies that Eq. (1) and/or Eq. (2) could perform well even in sufficiently intense turbulence. However, to the authors' knowledge, Eq. (2) has not yet been directly assessed by another research group for various species using DNS data associated with moderate or extreme turbulence. As far as a priori assessments of Eqs. (1), 
(3), and (4) are concerned, the present authors are aware on only two such tests, but they were restricted to either Eq. (1) applied to a single rate $\bar{W}_{c}$ [29] or Eq. (3) applied to the PDF $P(c)[25]$.

Recently, two of the present authors [33] quantitatively validated Eq. (2) for various species by analysing DNS data obtained by Dave and Chaudhuri [37] from a lean $\mathrm{H}_{2}$-air flame associated with thin reaction zone regime [38] of turbulent burning. Promising results reported in Ref. [33] for a single case require confirmation, a deeper analysis, and assessment for other regimes of premixed combustion.

The present work aims at filling the discussed knowledge gap by performing an a priori quantitative assessment of Eqs. (1), (2), and (3) for various species under conditions of weak, moderate, and intense turbulence. Note that the work is not limited to exploring these three equations all together, but it also aims at examining each equation separately. Indeed, the flamelet concept yielding Eqs. (1) and (2) and the presumed PDF approach, e.g. Eq. (3), are based on different reasoning and, therefore, Eqs. (1) and (2) could perform better than Eq. (3) or vice versa. Moreover, while both Eq. (1) and Eq. (2) stem from the same flamelet concept, the latter equation could perform better in a turbulent flow because variations in the mole fractions $X_{n}$ in a flame are smoother than variations in the rates $W_{n}$. Accordingly, eventual errors associated with the flamelet concept, i.e. reduction of $X_{n}(\mathbf{x}, t)$ and $W_{n}(\mathbf{x}, t)$ to $X_{n, L}[c(\mathbf{x}, t)]$ and $W_{n, L}[c(\mathbf{x}, t)]$, respectively, and eventual errors in modeling $P(c)$ could result in significantly larger errors when averaging the rates $W_{n}$ when compared to averaging the mole fractions $X_{n}$. In addition to the major goals stated above, i.e. examining Eqs. (1), (2), and (3) both jointly and separately, the present work aims also at assessing different choices of the combustion progress variable and the validity of the flamelet concept at high $K a$.

In the next section, the DNS data that are analyzed for these purposes are briefly summarized. The a priori analysis results are reported in Sect. 3, with their implications for modeling being discussed in Sect. 4. Last, conclusions are drawn in Sect. 5.

\section{Description of Simulation Data and Analysis}

Since the DNS computations were already discussed in detail elsewhere [39-43], only a brief summary of their major attributes is provided here. Freely-propagating, statistically one-dimensional and planar, lean (the equivalence ratio $F=0.7$ ) hydrogen-air turbulent flames were simulated by numerically integrating the unsteady three-dimensional governing equations in compressible form and using a detailed chemical mechanism [44] with 9 species and 19 reversible reactions. Under the simulation conditions (the unburned gas temperature $T_{u}=300 \mathrm{~K}$ and atmospheric pressure), the computed laminar flame speed $S_{L}=1.36 \mathrm{~m} / \mathrm{s}$ and thickness $\delta_{L}=\left(T_{b}-T_{u}\right) / \max |\nabla T|=0.36 \mathrm{~mm}$. The computed laminar flame profiles were adopted to initialize an initially planar flame.

To solve the governing equations, an eighth-order central difference and an explicit fourth-order Runge-Kutta schemes were used [40]. Inflow and outflow characteristic boundary conditions were utilized along the axis of the flame propagation while all other boundaries were set as periodic. A divergence-free, homogeneous, isotropic turbulence field was generated using a pseudo spectral method [45] following the Passot-Pouquet spectrum [46] and was injected through the inlet, with the mean inlet velocity being gradually changed to match the turbulent flame speed. The inflow values of the root-mean-square velocity $u^{\prime}$, the most energetic length scale $L_{T}$ of the Passot-Pouquet spectrum, Damköhler number $D a=L_{T} S_{L} /\left(u^{\prime} \delta_{L}\right)$, 
Karlovitz number $K a=\left(u^{\prime} / S_{L}\right)^{2} R e_{T}^{-1 / 2}\left(S_{L} \delta_{L} / v_{u}\right)$, and turbulent Reynolds number $R e_{T}=u^{\prime} L_{T} / v_{u}$ are listed in Table 1 . Here, $v_{u}$ is the molecular kinematic viscosity of unburned mixture. Based on the conditions at the inflow boundary, the simulated flames are associated with corrugated flamelet, thin reaction zone, and broken reaction zone regimes [38] in cases A, B, and C, respectively, although the effective $K a$ at the actual flame locations may be attenuated substantially [40].

Four different combustion progress variables are defined as follows: $c_{k}=\left(\phi_{k}-\phi_{k, u}\right) /\left(\phi_{k, b}-\phi_{k, u}\right)$, where subscripts $u$ and $b$ designate fresh reactants and equilibrium adiabatic combustion products, respectively, while $\phi_{1}, \phi_{2}$, and $\phi_{3}$ are the mass fractions of $\mathrm{H}_{2}, \mathrm{O}_{2}$, and $\mathrm{H}_{2} \mathrm{O}$, respectively, and $\phi_{4}$ is the temperature $T$.

The mean profiles $\bar{q}\left(\bar{c}_{k}\right)$ of various quantities $q$ are evaluated as follows. First, the $q(\mathbf{x}, t)$ and $c_{k}(\mathbf{x}, t)$-fields are averaged over the transverse plane ( $x=$ constant $)$ at each instant $t$ in order to calculate unsteady spatially averaged profiles of $\langle q\rangle(x, t)$ and $\left\langle c_{k}\right\rangle(x, t)$, respectively. Second, obtained dependencies of $\langle q\rangle(x, t)$ on $x$ are transformed to dependencies of $\langle q\rangle$ on a sample variable $\bar{\xi}$ using monotonic profiles of $\left\langle c_{k}\right\rangle(x, t)$ divided into 100 equal intervals, i.e. an $x$-plane contributes to the value of $\langle q\rangle\left(\bar{\xi}_{j}\right)$ provided that $\left|\left\langle c_{k}\right\rangle(x, t)-\bar{\xi}_{j}\right|<0.005$ with $\bar{\xi}=0.01 j$ and $j=0,1, \ldots, 100$. Subsequently, $\langle q\rangle(\bar{\xi}, t)$ is averaged over various instants in order to calculate mean profiles of $\bar{q}\left(\bar{c}_{k}\right)$ for each $c_{k}$.

Furthermore, to assess the predictive capabilities of the approach, both actual and $\beta$-function PDFs are determined and compared. The actual instantaneous PDFs $P_{k}(\xi, x, t)$ are sampled from grid points characterized by $\left|c_{k}(\mathbf{x}, t)-\xi_{j}\right|<0.002$ for each transverse plane at each instant. Here, $\xi=0.004 j$ with $j=0,1, \ldots, 250$ is a sample variable for the instantaneous $c_{k}(\mathbf{x}, t)$-fields. The actual PDFs are used not only as benchmarks when testing the $\beta$-function PDFs, but also for target-directed assessment of Eqs. (1) and (2) independently of Eqs. (3)-(4). Subsequently, the instantaneous actual PDFs $P_{k}(\xi, x, t)$ are transformed to $P_{k}(\xi, \bar{\xi}, t)$, as explained earlier, and are averaged over all instants to obtain the stationary PDFs $P_{k}\left(\xi, \bar{c}_{k}\right)$ for each $c_{k}$. The instantaneous $\beta$-function PDFs $P_{\beta, k}\left[\xi,\langle c\rangle_{k}(x, t),\left\langle c_{k}^{2}\right\rangle(x, t)\right]$ or $\left.\widetilde{P}_{\beta, k}[\xi, \widetilde{\langle c}\rangle_{k}(x, t),\left\langle\widetilde{c_{k}^{2}}\right\rangle(x, t)\right]$ are constructed using Eqs. (3) and (4) along with the profiles of $\langle c\rangle_{k}(x, t)$ and $\left\langle c_{k}^{2}\right\rangle(x, t)$ or $\widetilde{\langle c\rangle_{k}}(x, t)$ and $\left.\widetilde{c_{k}^{2}}\right\rangle(x, t)$, respectively, that are extracted from the DNS data at each instant, as discussed earlier. Similarly, the stationary $\beta$-function PDFs $P_{\beta, k}\left(\xi, \bar{c}_{k}\right)$ or $\bar{\rho}(\xi) \tilde{P}_{\beta, k}\left(\xi, \bar{c}_{k}\right) / \rho(\xi)$ are constructed using the actual stationary profiles of $\bar{c}_{k}(\bar{\xi})$ and $\overline{c_{k}^{2}}(\bar{\xi})$ or $\tilde{c}_{k}(\bar{\xi})$ and $\widetilde{c_{k}^{2}}(\bar{\xi})$, respectively. Finally, the mean profiles of $\bar{X}_{n}\left(\bar{c}_{k}\right), \bar{W}_{n}\left(\bar{c}_{k}\right), \bar{T}\left(\bar{c}_{k}\right)$, and $\bar{\rho}\left(\bar{c}_{k}\right)$ are calculated by adopting the stationary PDFs $P_{k}\left(\xi, \bar{c}_{k}\right)$, $P_{\beta, k}\left(\xi, \bar{c}_{k}\right)$, and $\bar{\rho}(\xi) \tilde{P}_{\beta, k}\left(\xi, \bar{c}_{k}\right) / \rho(\xi)$ to average the laminar flamelet library, i.e. a set of profiles of $X_{n, L}\left(c_{k}\right), W_{n, L}\left(c_{k}\right), T_{L}\left(c_{k}\right)$, and $\rho_{L}\left(c_{k}\right)$, respectively, that are pre-computed for a single lean hydrogen-air unperturbed laminar flame.

Mean profiles of $\bar{X}_{n}\left(\bar{c}_{k}\right), \bar{W}_{n}\left(\bar{c}_{k}\right), \bar{T}\left(\bar{c}_{k}\right)$, and $\bar{\rho}\left(\bar{c}_{k}\right)$, reported in the following were averaged over six different instants in each case, i.e., at $t / t_{e}=0.57,0.67,0.77,0.86,0.96$, and 1.05 in case $\mathrm{A}$ and $t / t_{e}=4.1,4.8,5.5,6.2,6.8$, and 7.5 in cases $\mathrm{B}$ and $\mathrm{C}$, with $t_{e}$ being the eddy turnover time. Results obtained at a single instant (in this case, statistics are directly extracted from the DNS data for each transverse plane) or averaged over several instants (less than six) show the same trends. 


\section{Results and Discussion}

Figure 1 reports results of assessment of Eq. (2), performed using four differently defined combustion progress variables separately (see dashed lines) and jointly with Eqs. (3) and (4) (see dotted and dotted-dashed lines) for major reactants and product. In cases A and B (see the left and middle columns) good agreement with the raw DNS data (see solid lines) is obtained adopting both the actual and $\beta$-function PDFs for each $c_{k}$. In case $\mathrm{C}$, the level of agreement decreases, but still appears to be acceptable, especially for $c_{4}=c_{T}$ and to a lesser extent for $c_{1}=c_{F}$ (i.e. when the combustion progress variable is defined using the temperature or fuel mass fraction, respectively, see the bottom and top panels, respectively, in the right column).

These results imply that case $\mathrm{C}$, characterized by $K a=126$, is close to the boundary of the validity domain for the flamelet concept, as far as the major reactants and product are concerned. Note, however, that the analyzed DNS data were obtained from lean hydrogen-air flames and, as reviewed elsewhere [47,48], differential-diffusion effects are well documented to play a significant role in turbulent combustion of lean $\mathrm{H}_{2}$-air flames. Such effects, in particular, result in changing the local flame structure when compared to the unperturbed laminar flame. Accordingly, the aforementioned boundary could be shifted to a higher $\mathrm{Ka}$ in lean or near-stoichiometric hydrocarbon-air flames, where differential-diffusion effects are less pronounced.

Note also that Fig. 1 is not a validation of the $\beta$-function PDF, but rather a confirmation that the averaged dependencies of $X_{n, L}\left(c_{k}\right)$ are weakly non-linear for $\mathrm{H}_{2}, \mathrm{O}_{2}$, and $\mathrm{H}_{2} \mathrm{O}$ (e.g. see the left panel in Fig. 6, which will be discussed later). Accordingly, any PDF that is based on the actual values of $\bar{c}$ and $\overline{c_{k}^{2}}$ should well predict the mean profiles of $\bar{X}_{n}\left(\bar{c}_{k}\right)$ for such species provided that the flamelet concept holds.

Figure 2 summarizes results of assessment of capabilities of the flamelet concept for predicting the mean density $\bar{\rho}$. For brevity, similar results computed for the mean temperature are not reported, because the density and temperature are simply coupled under isobaric conditions and a low Mach number. Major trends observed in Fig. 2 are similar to the already discussed trends shown in Fig. 1 with the following exception: in case C, the use of $c_{3}=c_{P}$ or $c_{4}=c_{T}$, defined based on the product $\left(\mathrm{H}_{2} \mathrm{O}\right)$ mass fraction or temperature, respectively, yields better results when compared to the other two $c_{k}$. Note also that the dependencies of $\bar{\rho}$ on $\overline{c_{k}}$ look linear in cases A and B, in line with the well-known BML equation $\bar{\rho}=\rho_{u}(1-\bar{c})+\rho_{b} \bar{c}[14]$, whereas such a linear relation does not hold in case $\mathrm{C}$.

Figure 3 displays results of assessment of Eq. (2) for radicals $\mathrm{H}, \mathrm{O}$, and $\mathrm{OH}$ (see black, blue, and red lines, respectively). The following trends are noted. First, if the combustion progress variable is defined based on the fuel mass fraction and the PDF is extracted from the DNS data (see the top row, cf. dashed and solid lines), the dependencies $\bar{X}_{n}\left(\bar{c}_{F}\right)$ are predicted with more satisfactory agreement for these three radicals in all three cases, despite a consistent underestimation of $\mathrm{OH}$ and an underestimation of $\mathrm{H}$ for the highly turbulent flame in case $\mathrm{C}$.

Second, the use of other $c_{k}$ yields substantially worse results (too low $\bar{X}_{n}$ adopting either the actual or a $\beta$-function PDF when compared to $\bar{X}_{n}$ extracted from the DNS data, cf. broken lines with solid lines) for each radical in each case, with the exception of $\bar{X}_{O H}\left(\bar{c}_{P}\right)$ and $\bar{X}_{O H}\left(\bar{c}_{T}\right)$ in case C. The present results computed for the radicals are in line with a recent analysis [49] of DNS data obtained from highly turbulent lean hydrogen-air flames, which have indicated that defining $c$ based on $\mathrm{H}_{2}$ 
is the best choice. However, the present study also supports the temperature-based $c_{T}$ as a valid choice as far as the mean density and mole fractions of $\mathrm{O}_{2}$ and $\mathrm{H}_{2} \mathrm{O}$ in the highly turbulent case $\mathrm{C}$ are concerned.

Third, better agreement between the raw DNS data and $\bar{X}_{n}\left(\bar{c}_{F}\right)$ is obtained when the PDF $P_{F}\left(\xi, \bar{c}_{F}\right)$ is extracted from the DNS data. The use of the $\beta$-function PDFs yields substantially underestimated mean radical mole fractions in cases A and B (see dotted or dotted-dashed lines) with the same trends being also observed for other $c_{k}$. These results clearly show substantial limitations of the $\beta$-function PDF at low and moderately large Karlovitz numbers under the conditions of the present study. However, in the highly turbulent case C, differences between $\bar{X}_{n}\left(\bar{c}_{k}\right)$ computed using the actual and $\beta$-function PDFs (cf. dashed and dotted or dotted-dashed lines, respectively) are less pronounced, thus suggesting that Eqs. (3) and (4) may perform better at large $K a$.

Comparison of the actual PDFs and PDFs yielded by Eqs. (3) and (4) confirms the above suggestion only in part. For instance, the actual $P_{F}\left(\xi, \bar{c}_{F}\right)$ and $\beta$-function $P_{\beta, F}\left(\xi, \bar{c}_{F}\right)$ PDFs obtained using $c_{F}$ in the middle of the flame brush $\left(\bar{c}_{F}=0.5\right)$ are shown in Fig. 4 in dotted and solid lines, respectively. On one hand, at $c_{F} \leq 0.8$, the two PDFs agree much better in case $\mathrm{C}$ than in case $\mathrm{A}$ or $\mathrm{B}$ (note also the large difference between the scales of the ordinate axes). The reader interested in fundamental flaws of the presumed PDF approach at low Karlovitz numbers is referred to a recent review paper [2]. On the other hand, even in case $\mathrm{C}$, the two $\mathrm{PDFs}^{1}$ are different at $\xi>0.8$, with the dependencies of $X_{n, L}\left(c_{F}\right)$ peaking at $c_{F}>0.8$ for $\mathrm{H}, \mathrm{O}$, and $\mathrm{OH}$ (see the left panel in Fig. 5). Due to these differences, the actual and $\beta$-function PDFs yield, respectively, $\bar{X}_{H}\left(\bar{c}_{F}=0.5\right)=0.09$ and $0.07, \bar{X}_{O}\left(\bar{c}_{F}=0.5\right)=0.031$ and 0.025 , and $\bar{X}_{O H}\left(\bar{c}_{F}=0.5\right)=0.029$ and 0.030 . The small difference in $\bar{X}_{O H}\left(\bar{c}_{F}=0.5\right)$ stems from (i) the closeness of the value of $c_{F}=0.95$ associated with the peak $X_{O H, L}\left(c_{F}\right)$, as seen in the left panel of Fig. 5, and the value of $\xi=0.93$ associated with the equality of the two PDFs (see the right panel in Fig. 4), and (ii) the symmetry of $X_{O H, L}\left(c_{F}\right)$ in the vicinity of its peak (see the left panel in Fig. 5). Accordingly, underestimation of the integral on the RHS of Eq. (2) at $0.8<\xi<0.93$ is roughly counterbalanced by overestimation of the integral at $\xi>0.93$.

Dashed lines in Fig. 4 show that, at $\xi<0.4$, the PDFs $\bar{\rho}\left(\bar{c}_{F}\right) \tilde{P}_{\beta, F}\left(\xi, \bar{c}_{F}\right) / \rho(\xi)$ agree with the actual PDFs better than the PDFs $P_{\beta, F}\left(\xi, \bar{c}_{F}\right)$ do. However, in a more interesting range of $\xi>0.4$, characterized by significantly larger radical concentrations and reaction rates (see Fig. 5), both $\bar{\rho}\left(\bar{c}_{F}\right) \tilde{P}_{\beta, F}\left(\xi, \bar{c}_{F}\right) / \rho(\xi)$ and $P_{\beta, F}\left(\xi, \bar{c}_{F}\right)$ differ significantly from the actual PDFs $P_{F}\left(\xi, \bar{c}_{F}\right)$, thus indicating limitations of the presumed $\beta$-function PDF approach.

All in all, Fig. 3 validates Eq. (2) in cases A and B provided that (i) the actual PDF is adopted and (ii) the combustion progress variable is defined using the mass fraction of the deficient reactant (fuel in the present work). Even in case C, the flamelet Eq. (2) yields encouraging results, despite a noticeable underprediction of $\mathrm{H}$.

These results are consistent with the profiles $\left\langle X_{n} \mid c_{F}\right\rangle\left(c_{F}\right)$ of the conditioned mole fractions of the radicals plotted in Fig. 6. In particular, the conditioned profiles are very close to the laminar-flame profiles $X_{n, L}\left(c_{F}\right)$ for $\mathrm{OH}$ and, especially, $\mathrm{O}$ in all three cases, while differences between $\left\langle X_{O H} \mid c_{F}\right\rangle\left(c_{F}\right)$ and $X_{O H, L}\left(c_{F}\right)$ are slightly increased from case A to case $\mathrm{C}$ (see the right

\footnotetext{
${ }^{1}$ In case $\mathrm{C}$, the actual PDF vanishes at large $c_{F}$, because (i) the PDF has been extracted from the middle of the mean flame brush $\left(\bar{c}_{F}=0.5\right)$ and (ii) the variance of the combustion progress variable is reduced with increasing $K a$.
} 
panel). Differences between $\left\langle X_{H} \mid c_{F}\right\rangle\left(c_{F}\right)$ and $X_{H, L}\left(c_{F}\right)$ (see the left panel) are more pronounced and increased from case A to case C, which shows a broader profile and higher peak. Nevertheless, there are some minor differences between Figs. 3 and 6. For instance, the left and middle panels in the first row in Fig. 3 indicate small differences between $\bar{X}_{H}\left(\bar{c}_{F}\right)$ calculated by substituting the actual PDF into Eq. (2) and $\bar{X}_{H}\left(\bar{c}_{F}\right)$ extracted from the DNS data (cf. dashed and solid lines, respectively), whereas the left panel in Fig. 6 shows noticeable differences between $\left\langle X_{H} \mid c_{F}\right\rangle\left(c_{F}\right)$ and $X_{H, L}\left(c_{F}\right)$ in cases A and B. This is because the conditioned profiles like $\left\langle X_{H} \mid c_{F}\right\rangle\left(c_{F}\right)$ are sampled from the entire flame brush, with each profile point corresponding to a particular value of $c_{F}$, whereas the mean profiles like $\bar{X}_{H}\left(\bar{c}_{F}\right)$ are sampled from a single transverse plane, involving contributions from various local $c_{F}(\mathbf{x}, t)$. Accordingly, differences in $\left\langle X_{n} \mid c_{F}\right\rangle\left(c_{F}, \bar{c}_{F}\right)$ and $X_{n, L}\left(c_{F}\right)$ at different $\bar{c}_{F}$ may counterbalance each other after integration over various $\bar{c}_{F}$. Conversely, differences in $\left\langle X_{n} \mid c_{F}\right\rangle\left(c_{F}, \bar{c}_{F}\right)$ and $X_{n, L}\left(c_{F}\right)$ could be significant at $\bar{c}_{F} \ll 1$ due to strong differential-diffusion effects in highly curved leading flamelets [47,48], thus, making $\left\langle X_{n} \mid c_{F}\right\rangle\left(c_{F}\right)$ and $X_{n, L}\left(c_{F}\right)$ noticeably different, but such effects are difficult to observe when reporting $\bar{X}_{n}\left(\bar{c}_{F}\right)$ for the entire range of $0 \leq \bar{c}_{F} \leq 1$. This point will be further addressed with the discussion of Fig. 10 .

Contrary to Eq. (2), the predictive capabilities of the flamelet Eq. (1) can be substantially worse, because variations in species concentrations in a flame are significantly smoother than variations in the rate of production/consumption of the same species in the same flame (cf. the left and right panels in Fig. 5). For instance, Fig. 7 shows that Eq. (1) poorly predicts the mean rate of production/consumption of radicals $\mathrm{H}$ and $\mathrm{O}$ even if the PDF is extracted from the DNS data and the weakly turbulent case A is considered.

Nevertheless, recall that Eq. (1) is commonly applied solely to evaluating the source term $\bar{W}_{c}$ in the transport equation for $\tilde{c}$ [15-32], whereas mean concentrations of various species are calculated using Eq. (2). Accordingly, the focus of assessment of performance of Eq. (1) should be placed on its ability to predict $\bar{W}_{c}$ for differently defined combustion progress variables. Such results are reported in Figs. 8 and 9 for species-based and temperature-based combustion progress variables, respectively. The following trends are noted.

First, substitution of the $\beta$-function PDFs $P_{\beta, k}\left(\xi, \bar{c}_{k}\right)$ or $\bar{\rho}\left(\bar{c}_{k}\right) \tilde{P}_{\beta, k}\left(\xi, \bar{c}_{k}\right) / \rho(\xi)$ given by Eqs. (3) and (4) into Eq. (1) (dotted or dotted-dashed lines, respectively) does not yield a good prediction of $\bar{W}_{c, k}$ (solid lines) for any $\bar{c}_{k}$ if cases A, B, and $\mathrm{C}$ are considered all together. Nevertheless, the mean heat release rate $\bar{W}_{T}\left(\bar{c}_{T}\right)$ is predicted sufficiently well adopting $\bar{\rho}\left(\bar{c}_{k}\right) \tilde{P}_{\beta, k}\left(\xi, \bar{c}_{k}\right) / \rho(\xi)$ in case A or $P_{\beta, k}\left(\xi, \bar{c}_{k}\right)$ in case C (cf. solid and dotted-dashed or dotted lines, respectively, in the left or right panel, respectively, in Fig. 8). Second, for the product-based combustion progress variable $c_{P}$ (red lines in Fig. 8), both the actual (dashed lines) and $\beta$-function PDFs (dotted and dotted-dashed lines) yield more comparable results in all three cases. The actual PDF clearly performs better for $c_{F}$ in case $\mathrm{A}$ or $c_{O}$ in cases $\mathrm{A}$ and $\mathrm{B}$, while differences between $\bar{W}_{c, k}\left(\bar{c}_{k}\right)$ obtained using various PDFs are least pronounced for each $\bar{c}_{k}$ in case C. Third, dependencies of $\bar{W}_{c, k}\left(\bar{c}_{k}\right)$ calculated by substituting the actual PDF into Eq. (1) differ substantially from dependencies of $\bar{W}_{c, k}\left(\bar{c}_{k}\right)$ extracted directly from the DNS data. The differences are least pronounced for the fuel-based $\bar{c}_{F}$ in case A (cf. blue solid and dashed lines in the left panel in Fig. 8). 
Conditioned rates of fuel consumption and heat release, plotted in the left and right panels in Fig. 10, respectively, also show that (i) differences between $\left\langle W_{F} \mid c_{F}\right\rangle\left(c_{F}\right)$ and $W_{F, L}\left(c_{F}\right)$ are the most (least) pronounced in case $\mathrm{C}$ (A, respectively), in line with Fig. 8, and (ii) differences between $\left\langle W_{T} \mid c_{T}\right\rangle\left(c_{T}\right)$ and $W_{T, L}\left(c_{T}\right)$ are more pronounced than the differences between $\left\langle W_{F} \mid c_{F}\right\rangle\left(c_{F}\right)$ and $W_{F, L}\left(c_{F}\right)$, in line with Figs. 8 and 9. Moreover, inspection of results reported in Figs. 8 and 10 indicates that flamelets are differently perturbed in different parts of the mean flame brush. For instance, comparison of dashed and solid blue lines in the left panel in Fig. 8 shows that Eq. (1) supplemented with the actual PDF slightly (less than 10\%) overestimates the magnitude of $\bar{W}_{F}$ in the largest part of the A-flame brush. However, the rate magnitude is significantly (more than 20\%) underestimated at low $\bar{c}_{F}$. Due to partial mutual cancellations of such deviations from the flamelet structure, $\left\langle W_{F} \mid c_{F}\right\rangle\left(c_{F}\right)$ and $W_{F, L}\left(c_{F}\right)$ are sufficiently close to one another in case A, cf. dotted and solid lines in the left panel in Fig. 10.

The considered differences between $\bar{W}_{k}\left(\bar{c}_{k}\right)$ extracted directly from the DNS data and $\bar{W}_{k}\left(\bar{c}_{k}\right)$ evaluated adopting Eq. (1) are consistent with substantial differences in the values of turbulent burning velocities $U_{T}$ obtained by integrating different instantaneous $\langle W\rangle_{c, k}\left[\langle c\rangle_{k}(x, t)\right]$ along the normal to the mean flame brush, i.e.,

$$
U_{T}(t)=\frac{M_{k}}{\rho_{u}\left(Y_{k, b}-Y_{k, u}\right)} \int_{-\infty}^{\infty}\langle W\rangle_{c, k}\left[\langle c\rangle_{k}(x, t)\right] d x
$$

for $\mathrm{H}_{2}(k=1), \mathrm{O}_{2}(k=2)$, and $\mathrm{H}_{2} \mathrm{O}(k=3)$, and

$$
U_{T}(t)=\frac{1}{\rho_{u}\left(T_{b}-T_{u}\right)} \int_{-\infty}^{\infty}\langle W\rangle_{c, 4}\left[\langle c\rangle_{4}(x, t)\right] d x
$$

if combustion progress variable is defined using the temperature $T$. Here, $\langle W\rangle_{c, 4}\left[\langle c\rangle_{4}(x, t)\right]$ is the instantaneous mean heat release rate expressed in $\mathrm{g} \cdot \mathrm{K} /\left(\mathrm{cm}^{3} \mathrm{~s}\right)$. As shown in Table 2, the values of the actual time-averaged burning velocity $\bar{U}_{T}$ differ substantially from $\bar{U}_{T}$ calculated using Eqs. (1) and (5) or (6) even if the instantaneous $\operatorname{PDF} P_{k}(\xi, \bar{\xi}, t)$ is directly extracted from the DNS data. For the fuel-based combustion progress variable, which yields the best results for the mean mole fractions of the radicals, $\bar{U}_{T}$ is overestimated by $7 \%$ in case A and $14 \%$ in case $\mathrm{B}$. In case C, the difference is small despite significant differences between the profiles of $\bar{W}_{F}\left(\bar{c}_{F}\right)$ extracted directly from the DNS data and calculated using Eq. (1), cf. solid and dashed blue lines in the right column in Fig. 8. Probably, the underestimation of $\left|\bar{W}_{F}\right|\left(\bar{c}_{F}\right)$ at $\bar{c}_{F}<0.5$ is occasionally counterbalanced by the overestimation of $\left|\bar{W}_{F}\right|\left(\bar{c}_{F}\right)$ at $\bar{c}_{F}>0.6$ in this case.

Note that substitution of the actual PDF with a presumed $\beta$-function PDF involves an extra source of error and, accordingly, is expected to perform worse. This is indeed observed in some cases. For the fuel-based combustion progress variable in case $\mathrm{A}$, the mean burning velocity $\bar{U}_{T}$ is overestimated by about $27 \% \quad(56 \%)$ if the presumed mass-weighted (conventional, respectively) $\beta$-function PDF is invoked even if the PDF is modeled adopting the first two moments of the $c_{F}(\mathbf{x}, t)$-field extracted from the DNS data. The effect magnitude can be significantly larger, e.g. $\bar{U}_{T}$ is underestimated by a factor of about five when using the oxygen-based $c_{O}$ in case A. Nevertheless, in some cases (e.g. see the dependencies of $\bar{W}_{T}\left(\bar{c}_{T}\right)$ shown in Fig. 9 or the values of $\bar{U}_{T}$ obtained adopting $\tilde{P}_{\beta, T}(\xi, \bar{\xi}, t)$ in case B or $P_{\beta, O}(\xi, \bar{\xi}, t), P_{\beta, P}(\xi, \bar{\xi}, t)$, and 
$P_{\beta, T}(\xi, \bar{\xi}, t)$ in case $\left.\mathrm{C}\right)$, errors due to the use of the flamelet library for the reaction rates and errors due to the use of the $\beta$ function PDF occasionally counterbalance one another and mislead the validity and accuracy of Eqs. (1), (3), and (4).

Note also that $\bar{U}_{T}$ computed in case $\mathrm{C}$ characterized by the highest $u^{\prime} / S_{L}$ and $K a$ is lower than $\bar{U}_{T}$ found in case B. This difference may be attributed, at least in part, to a low ratio of $L_{T} / \delta_{L}$ in case $\mathrm{C}$, as turbulent burning velocity is increased by $L_{T} / \delta_{L}[50,51]$ and is proportional to $\left(u^{\prime} L_{T} S_{L} / \delta_{L}\right)^{1 / 2}$ at high Karlovitz numbers [52-54]. At the same time, the peak values of the $\bar{W}_{c, k}\left[\bar{c}_{k}(x)\right]$ show the opposite trend, i.e. the mean rates are larger in case $\mathrm{C}$ when compared to case $\mathrm{B}$. The point is that the computed mean flame brush thickness is significantly smaller in case $\mathrm{C}$, thus, yielding a smaller integral on the right hand side of Eq. (5) or (6).

\section{Implications for Modeling}

The present analysis of DNS data quantitatively validates the flamelet Eq. (2) for major reactants, products, and radicals $\mathrm{H}, \mathrm{O}$, and $\mathrm{OH}$ even in the highly turbulent case $\mathrm{C}$ (albeit with slightly reduced accuracy), but does not support the presumed $\beta$-function PDF approach, i.e. Eqs. (3) and (4). Moreover, the DNS data show that the use of Eq. (1) yields substantial errors in determining (i) the source term $\bar{W}_{c, k}$ in the transport equation for the mean combustion progress variable $\bar{c}_{k}$ and, consequently, (ii) turbulent burning velocity. Such errors are significant even if the PDF is directly extracted from the DNS data. These results imply that even for lean hydrogen-air mixtures, the flamelet concept could be useful at Karlovitz numbers on the order of 100. However, to utilize the potential of the concept, Eq. (2) should be adopted independently of Eq. (1). More specifically, instead of using Eq. (1) to close the source term $\bar{W}_{c}$ in the transport equation for a mean combustion progress variable, Eq. (2) could be utilized to evaluate mean mole fractions of various species at a post-processing stage after computations were done by invoking another model of $\bar{W}_{c}$, which performed better than Eq. (1) in predicting the mean flame speed, thickness, and structure. Such a model could be sufficiently simple and use complex-chemistry data solely in a form of the laminar flame speed and thickness.

Discussion of such a closure relation is beyond the scope of the present work and the interested reader is referred to recent books [48,55-57] and a review paper [58]. Here, we restrict ourselves to noting that predictive capabilities of such "single-stepchemistry" models should not be underestimated. Indeed, first, as hypothesized by Prudnikov [59], reviewed elsewhere [48,50], and supported by more recent experimental data [60,61], various premixed turbulent flames have the same mean structure, i.e., variations of the mean $\bar{c}$ along the normal to a mean flame surface are well approximated by the complementary error function if the distance is normalized with the mean flame brush thickness. Single-step-chemistry models can predict this fundamental feature of premixed turbulent combustion [48,50,62-64], whereas any effect of combustion chemistry on the mean flame structure has not yet been revealed.

Second, as hypothesized by Karlovitz et al. [65], reviewed elsewhere [48,50,59] and supported by more recent data $[61,66]$, the growth of mean turbulent flame brush thickness follows the turbulent diffusion law in various experiments. Singlestep-chemistry models can predict this fundamental feature of premixed turbulent combustion $[48,50,64]$, whereas any effect of combustion chemistry on the growth of mean flame brush thickness has not yet been documented. 
Third, while some influence of combustion chemistry on burning rate is expected due to local extinction phenomena in extreme turbulence, the flamelet concept does not seem to hold under such conditions. As far as less, but still intense turbulence is concerned, single-step-chemistry models can predict burning rate reasonably well, as reviewed elsewhere [48,50,64]. For instance, in recent DNS studies [67,68], weak influence of combustion chemistry on turbulent burning velocity was documented at Karlovitz numbers significantly higher than unity.

To apply Eq. (2) at a post-processing stage, not only a closure relation for the mean rate $\bar{W}_{c}$, but also a PDF $P(c, \mathbf{x}, t)$ are required, and modeling the PDF still challenges the combustion community. The issue could be addressed by employing an approach that deals with a transport equation for the PDF [6-9]. This research direction appears to be prioritized from the fundamental perspective and the present results provide additional motivation for such a study.

Alternatively, from the application perspective, the presumed PDF approach could be further developed. In particular, first, if Eq. (1) is not used, but another model of $\bar{W}_{c}$ is invoked, mean concentrations of various species could be evaluated at the post-processing stage by adopting a presumed PDF that involves one more unknown parameter. Indeed, in such a case, the following three equations

$$
\begin{gathered}
1=\int_{0}^{1} P(c, \mathbf{x}, t) d c \\
\bar{c}(\mathbf{x}, t)=\int_{0}^{1} c P(c, \mathbf{x}, t) d c \\
\overline{c^{2}}(\mathbf{x}, t)=\int_{0}^{1} c^{2} P(c, \mathbf{x}, t) d c
\end{gathered}
$$

which are typically applied to evaluate parameters of a common presumed PDF $P(c, \mathbf{x}, t)$, could be supplemented with one more constraint of

$$
\bar{W}_{c}(\mathbf{x}, t)=\int_{0}^{1} W_{c}(c) P(c, \mathbf{x}, t) d c
$$

with the mean rate on the left hand side being provided by the invoked model of $\bar{W}_{c}$. The use of this extra calibration constraint will offer an opportunity to consider a wider set of presumed PDFs.

Alternatively, Eq. (9) could be substituted with Eq. (10). In such a case, the number of the PDF parameters remain at three, but a transport equation for $\overline{c^{\prime \prime 2}}(\mathbf{x}, t)$ or $\widetilde{c^{\pi / 2}}(\mathbf{x}, t)$ needs not be solved, thus, circumventing the issue of closure models for the scalar dissipation rate appearing in that transport equation [57]. Moreover, substitution of Eq. (9) with Eq. (10) offers an opportunity to obtain a PDF that predicts the probability of finding reaction zones significantly better, because the rate $W_{c}$ is low outside the reaction zones and, hence, the mean rate $\bar{W}_{c}$ is more closely correlated with that probability, whereas the variance $\overline{c^{\prime \prime 2}}(\mathbf{x}, t)$ or $\widetilde{c^{\prime \prime 2}}(\mathbf{x}, t)$ is not necessarily correlated with the probability referred to [2,48]. The latter claim is obvious in the BML limit [14], where these variances are solely controlled by the probabilities of finding unburned reactants and equilibrium combustion products. Furthermore, the mean rate $\bar{W}_{c}(\mathbf{x}, t)$ implicitly contains information on all moments of the $c(\mathbf{x}, t)$-field, whereas a common presumed PDF is built by adopting the first two moments only. For this basic reason, 
substitution of Eq. (9) with Eq. (10) for calibrating a presumed PDF has a potential to yield a more accurate $P(c)$. In summary, substitution of Eq. (9) with Eq. (10) allows us

- to circumvent a closure relation for the mean scalar dissipation rate,

- $\quad$ to better evaluate the probability of finding reaction zones, and

- $\quad$ to allow implicitly for all moments of the $c(\mathbf{x}, t)$-field.

Since the use of Eq. (10) for calibrating a presumed PDF requires a high-fidelity closure relation for the mean rate $\bar{W}_{c}$, predictive capabilities of the outlined approach are limited by the selected model of $\bar{W}_{c}$. Nevertheless, as discussed in detail elsewhere $[48,55-58,64]$, there are sufficiently mature turbulent combustion models that do not use Eq. (1) to close $\bar{W}_{c}$. Therefore, predictions obtained by invoking such a model are expected to be improved when compared to results computed by adopting Eq. (1) to close $\bar{W}_{c}$. Moreover, for the basic reasons discussed above, the joint use of such a model and Eq. (10) to calibrate $P(c)$ is superior to the calibration of $P(c)$ with Eq. (9).

While calibration of a presumed PDF by solving Eq. (10) may result in additional errors when compared to using simple algebraic relations given by Eq. (4), the latter relations hold for the $\beta$-function PDF only, but this PDF performs poorly under conditions of the present study. If another shape of the presumed $P(c)$ is invoked, either Eq. (9) or Eq. (10) should be solved to calibrate the PDF. If the integrals in these equations are pre-tabulated using a flamelet library, substitution of Eq. (9) with Eq. (10) is not expected to significantly increase the simulation costs.

The encouraging results obtained in the present work by examining the flamelet Eq. (2) suggest that the presumed PDF approach is worth advancing based on the flamelet PDF, i.e. $1 /\left(\delta_{L}|\nabla c|_{L}\right)[11,12]$. Development and assessment of such a presumed flamelet-based PDF will be a subject for a future study, in addition to further analysis of the DNS data [39-43] as well as other complex-chemistry DNS data.

\section{Conclusions}

A quantitative a priori assessment of the flamelet approach to evaluating mean concentrations of various species in a premixed turbulent flame has been performed by analysing complex-chemistry DNS data obtained earlier [39-43] from three lean hydrogen-air flames associated with corrugated flame (case A, $K a=0.75$ ), thin reaction zone (case B, $K a=14.4$ ), and broken reaction zone (case $\mathrm{C}, \mathrm{Ka}=126$ ) regimes of premixed turbulent burning. The approach consists in (i) simulating the unperturbed laminar flame in order to obtain dependencies of the temperature, density, mole fractions and rates of production/consumption of various species on a single combustion progress variable $c$ and (ii) averaging these dependencies by invoking a PDF for the same combustion progress variable. When assessing the approach, four different choices of $c$ have been probed and the PDF (i) either has been extracted directly from the DNS data or (ii) has been modelled invoking the wellknown presumed $\beta$-function PDF and using the first two moments of the $c$-field yielded by the DNS data.

The results show that, not only in case A, but also in case $\mathrm{B}$, and even in case $\mathrm{C}$, the mean density and the mean mole fractions of $\mathrm{H}_{2}, \mathrm{O}_{2}$, and $\mathrm{H}_{2} \mathrm{O}$ are satisfactorily predicted by Eq. (2) adopting both PDFs for each $c_{k}$, although the predictive capabilities of Eq. (2) worsen in case C. In cases A and B, the use of the actual PDF and the fuel-based combustion progress 
variable also offers an opportunity to well predict mean mole fractions of radicals $\mathrm{O}$ and $\mathrm{H}$, whereas the mean mole fraction of $\mathrm{OH}$ is slightly underestimated. In the highly turbulence case C, Eq. (2) still appears to be acceptable for evaluating the mean radical concentrations while the mean mole fraction of $\mathrm{H}$ is moderately underestimated. These results indicate that the flamelet concept may be useful even for lean hydrogen-air mixtures at Karlovitz numbers on the order of 100 .

The use of another (oxygen, product, or temperature-based) combustion progress variable or the $\beta$-function PDFs yields worse results for the aforementioned radicals. Direct comparison of the actual and $\beta$-function PDFs also shows substantial limitations of the latter model.

When compared to the mean mole fractions, the mean rate of product creation, i.e. the source term in the transport equation for $\bar{c}$, and, consequently, turbulent burning velocity are substantially worse predicted by Eq. (1) using the actual PDF extracted from the DNS data or a $\beta$-function PDF, which performs poorly in cases A and B. Accordingly, while both Eq. (1) and (2) are based on the flamelet concept, their predictive capabilities are substantially different, i.e. predictive capabilities of Eq. (2) are significantly better. Therefore, Eq. (2) is worth coupling with another model of premixed turbulent combustion whose predictive capabilities are better documented when compared to Eq. (1). In such a case, Eq. (2) could be implemented as postprocessing of a mean $\bar{c}$-field computed by numerically integrating a transport equation for the mean combustion progress variable.

All in all, the flamelet-based Eq. (2) appears to hold under various conditions ranging from weakly turbulent combustion (case A) to highly turbulent burning (case B and even case C) provided that an appropriate definition of combustion progress variable is adopted and the PDF $P(c)$ is well modeled. Therefore, these results (i) indicate that the domain of the flamelet concept validity is substantially wider than it was earlier assumed, in line with recent studies [36,54], and (ii) motivate improved modeling of the PDF in Eq. (2). Extension of the present work to filtered scalar fields and filtered density functions computed in a LES could also be a subject for future studies.

\section{Acknowledgements}

ANL gratefully acknowledges the financial support provided by CERC. VAS gratefully acknowledges the financial support provided by ONERA and by the Grant of the Ministry of Education and Science of the Russian Federation (Contract No. 14.G39.31.0001 of 13.02.2017). WS, FEHP, and HGI were sponsored by King Abdullah University of Science and Technology (KAUST). Computational resources for the DNS calculations were provided by the KAUST Supercomputing Laboratory.

\section{References}

[1] L.Y.M. Gicquel, G. Staffelbach, T. Poinsot, Large Eddy Simulations of gaseous flames in gas turbine combustion chambers, Prog. Energy Combust. Sci. 38(6) (2012) 782-815.

[2] A.N. Lipatnikov, Stratified turbulent flames: Recent advances in understanding the influence of mixture inhomogeneities on premixed combustion and modeling challenges, Prog. Energy Combust. Sci. 62 (2017) 87-132.

[3] B. Fiorina, R. Vicquelin, P. Auzillon, N. Darabiha, O. Gicquel, D. Veynante, A filtered tabulated chemistry model for LES of premixed combustion, Combust. Flame 157 (2010) 465-475.

[4] O. Gicquel, N. Darabiha, D. Thévenin, Laminar premixed hydrogen/air counterflow flame simulations using flame prolongation of ILDM with differential diffusion, Proc. Combust. Inst. 28 (2000) 1901-1908.

[5] J.A. van Oijen, L.P.H. de Goey, Modeling of premixed laminar flames using flamelet generated manifolds, Combust. Sci. Technol. 161 (2000) 113-137.

[6] E.E. O’Brien, in P.A. Libby, F.A. Williams (Eds.), Turbulent Reacting Flows, Springer-Verlag, Berlin, 1980, p. 185.

[7] S.B. Pope, PDF methods for turbulent reactive flows, Prog. Energy Combust. Sci. 11 (1985) 119-192. 
[8] C. Dopazo, in P.A. Libby, F.A. Williams (Eds.), Turbulent Reacting Flows, Academic Press, London, 1994, p. 375.

[9] D.C. Haworth, Progress in probability density function methods for turbulent reacting flows, Prog. Energy Combust. Sci. 36 (2010) 168-259.

[10] P.A. Libby, F.A. Williams, Presumed PDF analysis of partially premixed turbulent combustion, Combust. Sci. Technol. 161 (2000) 359-390.

[11] K.N.C. Bray, M. Champion, P.A. Libby, N. Swaminathan, Finite rate chemistry and presumed PDF models for premixed turbulent combustion, Combust Flame 146 (2006) 665-673.

[12] M.M. Salehi, W.K. Bushe, N. Shahbazian, C.R.T. Groth, Modified laminar flamelet presumed probability density function for LES of premixed turbulent combustion, Proc. Combust. Inst. 34 (2013) 1203-1211.

[13] N. Peters, The premixed turbulent flame in the limit of a large activation energy, J. Non-Equilibrium Thermodynamics 7 (1982) 25-38

[14] K.N.C. Bray, P.A. Libby, J.B. Moss, Unified modeling approach for premixed turbulent combustion - Part I: General formulation, Combust. Flame 61 (1985) 87-102.

[15] B. Fiorina, O. Gicquel, L. Vervisch, S. Carpentier, N. Darabiha, Premixed turbulent combustion modeling using tabulated detailed chemistry and PDF, Proc. Combust. Inst. 30 (2005) 867-874.

[16] A.W. Vreman, J.A. van Oijen, L.P.H. de Goey, R.J.M. Bastiaans, Subgrid scale modeling in large-eddy simulation of turbulent combustion using premixed flamelet chemistry, Flow Turbul. Combust. 82 (2009) 511-535.

[17] H. Kolla, N. Swaminathan, Strained flamelets for turbulent premixed flames II: Laboratory flame results, Combust. Flame, 157 (2010) 1274-1289.

[18] P. Trisjono, K. Kleinheinz, S. Kang, H. Pitsch, Large eddy simulation of stratified and sheared flames of a premixed turbulent stratified flame burner using a flamelet model with heat loss, Flow Turbul. Combust. 92 (2014) 201-235.

[19] F. Hernández-Pérez, C. Groth, Ö. Gülder, Large-eddy simulation of lean hydrogen-methane turbulent premixed flames in the methane-dominated regime, Int. J. Hydrogen Energy 39 (2014) 7147-7157.

[20] F. Hernández-Pérez, F. Yuen, C. Groth, Ö. Gülder, LES of a laboratory-scale turbulent premixed Bunsen flame using FSD, PCM-FPI and thickened flame models, Proc. Combust. Inst. 33 (2011) 1365-1371.

[21] J. Galpin, A. Naudin, L. Vervisch, C. Angelberger, O. Colin, P. Domingo, Large-eddy simulation of a fuel-lean premixed turbulent swirl-burner, Combust. Flame 155 (2008) 247-266.

[22] O.R. Darbyshire, N. Swaminathan, A presumed joint PDF model for turbulent combustion with varying equivalence ratio, Combust. Sci. Technol. 184 (2012) 2036-2067.

[23] S. Nambully, P. Domingo, V. Moreau, L. Vervisch, A filtered-laminar-flame PDF sub-grid scale closure for LES of premixed turbulent flames: I. Formalism and application to a bluff-body burner with differential diffusion, Combust. Flame 161 (2014) 1756-1774.

[24] S. Nambully, P. Domingo, V. Moreau, L. Vervisch, A filtered-laminar-flame PDF sub-grid scale closure for LES of premixed turbulent flames: II. Application to a stratified bluff-body burner, Combust. Flame 161 (2014) 1775-1791.

[25] A. Donini, R.J.M. Bastiaans, J.A. van Oijen, M.S. Day, L.P.H. de Goey, An a priori DNS subgrid analysis of the presumed $\beta$-PDF model, Int. J. Hydrogen Energy 40 (2015) 12811-12823.

[26] J.A. van Oijen, A. Donini, R.J.M. Bastiaans, J.H.M. ten Thije Boonkkamp, L.P.H. de Goey, State-of-the-art in premixed combustion modeling using flamelet generated manifolds, Prog. Energy Combust. Sci. 57 (2016) 30-74.

[27] I. Langella, N. Swaminathan, Unstrained and strained flamelets for LES of premixed combustion, Combust. Theory Modelling 20 (2016) 410-440.

[28] I. Langella, N. Swaminathan, R.W. Pitz, Application of unstrained flamelet SGS closure for multiregime premixed combustion, Combust. Flame 173 (2016) 161-178.

[29] S. Lapointe, G. Blanquart, A priori filtered chemical source term modeling for LES of high Karlovitz number premixed flames, Combust. Flame 176 (2017) 500-510.

[30] A. Donini, R.J.M. Bastiaans, J.A. van Oijen, L.P.H. de Goey, A 5-D implementation of FGM for the large eddy simulation of a stratified swirled flame with heat loss in a gas turbine combustor, Flow Turbul. Combust. 98 (2017) 887-922.

[31] F.C.C. Galeazzo, B. Savard, H. Wang, E.R. Hawkes, J.H. Chen, G.C.K. Filho, Performance assessment of flamelet models in flame-resolved LES of a high Karlovitz methane/air stratified premixed jet flame, Proc. Combust. Inst. 37 (2019) 25452553.

[32] T. Nilsson, R. Yu, N.A.K. Doan, I. Langella, N. Swaminathan, X. Bai, Filtered reaction rate modelling in moderate and high Karlovitz number flames: an a prior analysis, Flow Turbul. Combust. 103 (2019) 643-665.

[33] A.N. Lipatnikov, V.A. Sabelnikov, Evaluation of mean species mass fractions in premixed turbulent flames: A DNS study, Proc. Combust. Inst. 38 (2021), available online https://doi.org/10.1016/j.proci.2020.05.006

[34] A.N. Lipatnikov, J. Chomiak, Effects of premixed flames on turbulence and turbulent scalar transport, Prog. Energy Combust. Sci. 36 (2010) 1-102.

[35] V.A. Sabelnikov, A.N. Lipatnikov, Recent advances in understanding of thermal expansion effects in premixed turbulent flames, Annu. Rev. Fluid Mech. 49 (2017) 91-117.

[36] J.F. Driscoll, J.H.Chen, A.W.Skiba, C.D.Carter, E.R.Hawkes, H. Wang, Premixed flames subjected to extreme turbulence: Some questions and recent answers, Prog. Energy Combust. Sci. 76 (2020) 100802.

[37] H. Dave, S. Chaudhuri, Evolution of local flame displacement speeds in turbulence, J. Fluid Mech. 884 (2020) A46.

[38] N. Peters, Turbulent Combustion, Cambridge University Press, Cambridge, 2000.

[39] H.A. Uranakara, S. Chaudhuri, H.L. Dave, P.G. Arias, H.G. Im, A flame particle tracking analysis of turbulence-chemistry interaction in hydrogen-air premixed flames, Combust. Flame 163 (2016) 220-240.

[40] H.G. Im, P.G. Arias, S. Chaudhuri, H.A. Uranakara, Direct numerical simulations of statistically stationary turbulent premixed flames, Combust. Sci. Technol. 188 (2016) 1182-1198.

[41] D.H. Wacks, N. Chakraborty, M. Klein, P.G. Arias, H.G. Im, Flow topologies in different regimes of premixed turbulent combustion: A direct numerical simulation analysis, Phys. Rev. Fluids 1 (2016) 083401. 
[42] D.M. Manias, E.Al. Tingas, F.E. Hernández Pérez, R.M. Galassi, P.P. Ciottoli, M. Valorani, H.G. Im, Investigation of the turbulent flame structure and topology at different Karlovitz numbers using the tangential stretching rate index, Combust. Flame 200 (2019) 155-167.

[43] M. Klein, A. Herbert, H. Kosaka, B. Böhm, A. Dreizler, N. Chakraborty, V. Papapostolou, H.G. Im, J. Hasslberger, Evaluation of flame area based on detailed chemistry DNS of premixed turbulent hydrogen-air flames in different regimes of combustion, Flow Turbul. Combust. 104 (2020) 403-419.

[44] M.P. Burke, M. Chaos, Y. Ju, F.L. Dryer, S.J. Klippenstein, Comprehensive $\mathrm{H}_{2} / \mathrm{O}_{2}$ kinetic model for high-pressure combustion, Int. J. Chem. Kinet. 44 (2012) 444-474.

[45] R.S. Rogallo, Numerical Experiments in Homogeneous Turbulence, NASA Technical Memorandum 81315, NASA Ames Research Center, California, 1981.

[46] T. Passot, A. Pouquet, Numerical simulation of compressible homogeneous flows in the turbulent regime, J. Fluid Mech. 181 (1987) 441-466.

[47] A.N. Lipatnikov, Molecular transport effects on turbulent flame propagation and structure, Prog. Energy Combust. Sci. 31 (2005) 1-73.

[48] A.N. Lipatnikov, Fundamentals of Premixed Turbulent Combustion, CRC Press, Boca Raton, Florida, 2012.

[49] M. Kuron, E.R. Hawkes, Z. Ren, J.C.K. Tang, H. Zhou, J.H. Chen, T. Lu, Performance of transported PDF mixing models in a turbulent premixed flame, Proc. Combust. Inst. 36 (2017) 1987-1995.

[50] A.N. Lipatnikov, J. Chomiak, Turbulent flame speed and thickness: Phenomenology, evaluation, and application in multidimensional simulations, Prog. Energy Combust. Sci. 28 (2002) 1-73.

[51] R. Yu, A.N. Lipatnikov, Direct numerical simulation study of statistically stationary propagation of a reaction wave in homogeneous turbulence, Phys. Rev. E 95 (2017) 063101.

[52] R. Yu, A.N. Lipatnikov, DNS study of dependence of bulk consumption velocity in a constant-density reacting flow on turbulence and mixture characteristics, Phys. Fluids 29 (2017) 065116.

[53] A.J. Aspden, M.J. Day, J.B. Bell, Towards the distributed burning regime in turbulent premixed flames, J. Fluid Mech. $871(2019) 1-21$.

[54] V.A. Sabelnikov, R. Yu, A.N. Lipatnikov, Thin reaction zones in constant-density turbulent flows at low Damköhler numbers: Theory and simulations, Phys. Fluids 31 (2019) 055104.

[55] T. Poinsot, D. Veynante, Theoretical and Numerical Combustion, 2nd ed., Edwards, Philadelphia, 2005.

[56] T. Echekki, E. Mastorakos (Eds.), Turbulent Combustion Modeling, Springer, Berlin, 2011.

[57] N. Swaminathan, K.N.C. Bray (Eds.), Turbulent Premixed Flames, Cambridge University Press, Cambridge, U.K., 2011.

[58] R.W. Bilger, S.B. Pope, K.N.C. Bray, J.F. Driscoll, Premixed flames subjected to extreme turbulence: Some questions and recent answers, Proc. Combust. Inst. 30 (2005) 21-42

[59] A.G. Prudnikov, in B.V. Raushenbakh (Ed.), Physical Principles of the Working Process in Combustion Chambers of Jet Engines, Clearing House for Federal Scientific \& Technical Information, Springfield, 1967, p. 244.

[60] P. Tamadonfar, Ö.L. Gülder, Flame brush characteristics and burning velocities of premixed turbulent methane/air Bunsen flames, Combust. Flame 161 (2014) 3154-3165.

[61] D. Han, A. Satija, J.P. Gore, R.P. Lucht, Experimental study of $\mathrm{CO}_{2}$ diluted, piloted, turbulent $\mathrm{CH}_{4} /$ air premixed flames using high-repetition-rate OH PLIF, Combust. Flame 193 (2018) 145-156.

[62] A.N. Lipatnikov, J. Chomiak, A theoretical study of premixed turbulent flame development, Proc. Combust. Inst. 30 (2005) 843-850.

[63] A.N. Lipatnikov, J. Chomiak, Self-similarly developing, premixed, turbulent flames: a theoretical study, Phys. Fluids 17 (2005) 065105.

[64] A.N. Lipatnikov, in S. De, A.K. Agarwal, S. Chaudhuri, S. Sen (Eds.), Modeling and Simulation of Turbulent Combustion, Springer Nature Singapore Pte Ltd., 2018, p. 181.

[65] B. Karlovitz, D.W. Denniston, F.E. Wells, Investigation of turbulent flames, J. Chem. Phys. 19 (1951) 541-547.

[66] T. Sponfeldner, N. Soulopoulos, F. Beyrau, Y. Hardalupas, A.M.K.P. Taylor, J.C. Vassilicos, The structure of turbulent flames in fractal- and regular-grid-generated turbulence, Combust. Flame 162 (2015) 3379-3393.

[67] S. Lapointe, G. Blanquart, Fuel and chemistry effects in high Karlovitz premixed turbulent flames, Combust. Flame 167 (2016) 294-307.

[68] Z. Wang, V. Magi, J. Abraham, Turbulent flame speed dependencies of lean methane-air mixtures under engine relevant conditions, Combust Flame 180 (2017) 53-62. 
FIGURES

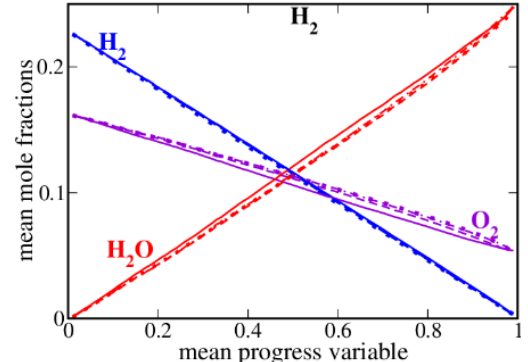

(a)

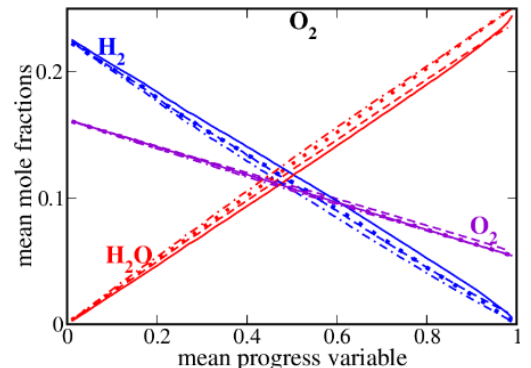

(d)

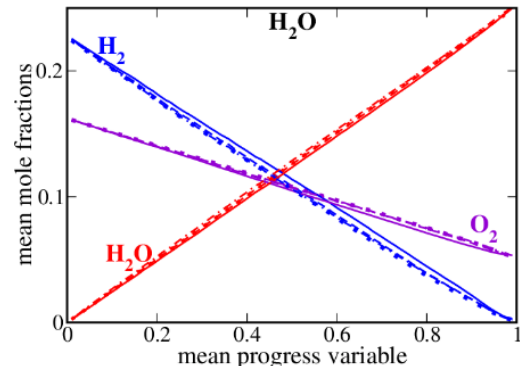

(g)

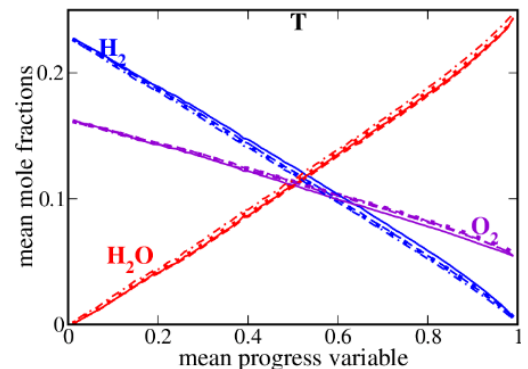

(j)

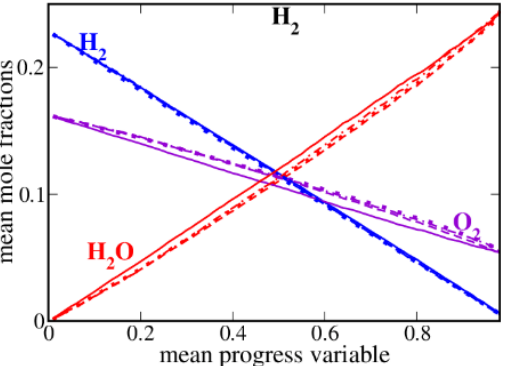

(b)

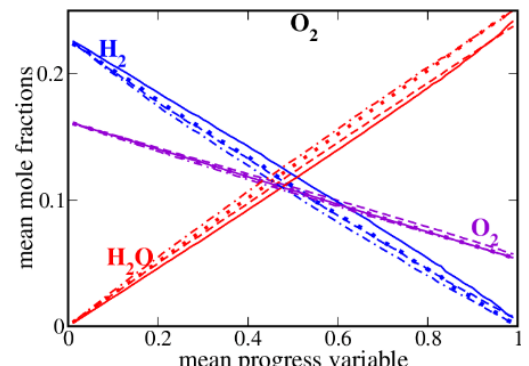

(e)

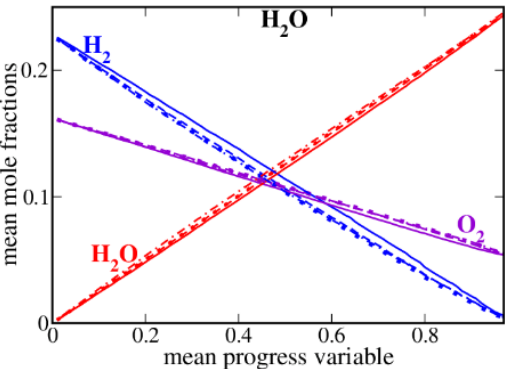

(h)

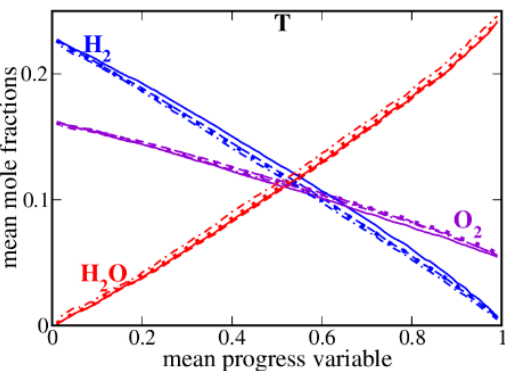

(k)

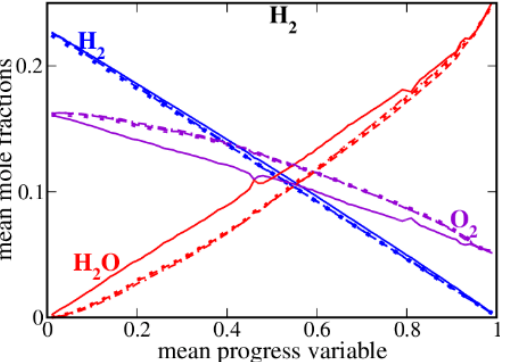

(c)

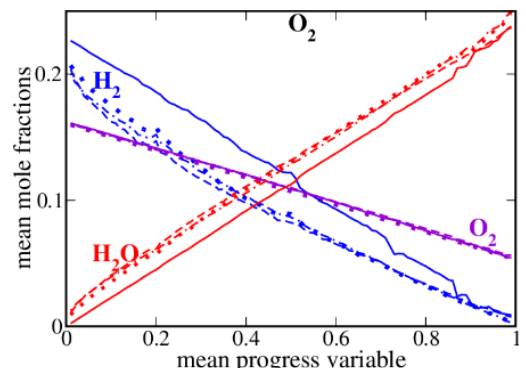

(f)

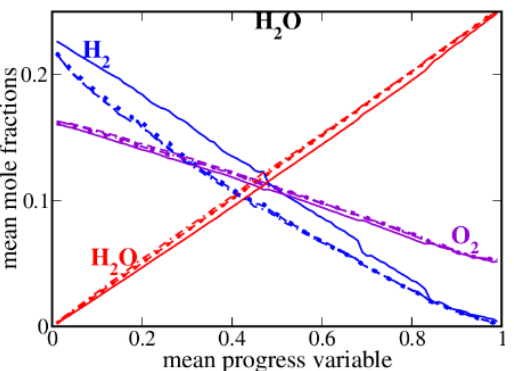

(i)

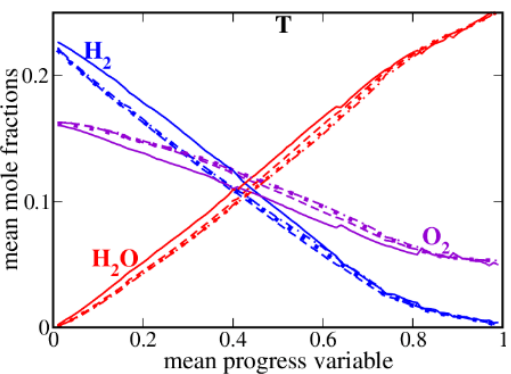

(l)

Fig. 1. Mean mole fractions $\bar{X}_{n}$ of major reactants and products noted near relevant curves vs. differently defined mean combustion progress variables $\bar{c}_{k}$. Results obtained adopting mass fractions of $\mathrm{H}_{2}, \mathrm{O}_{2}, \mathrm{H}_{2} \mathrm{O}$, and temperature $T$ to define $c_{k}$ are shown in the first, second, third, and fourth rows, respectively. Results obtained from flames A, B, and C are plotted in the left, middle, and right columns, respectively. Solid lines show $\bar{X}_{n}\left(\bar{c}_{k}\right)$ extracted directly from the DNS data. Dashed lines show $\bar{X}_{n}\left(\bar{c}_{k}\right)$ evaluated using the laminar-flame profiles of $X_{n, L}\left(c_{k}\right)$ and the actual PDFs $P_{k}\left(\xi, \bar{c}_{k}\right)$ extracted from the DNS data. Dotted and dotted-dashed lines show $\bar{X}_{n}\left(\bar{c}_{k}\right)$ calculated adopting the $\beta$-function PDFs $P_{\beta, k}\left(\xi, \bar{c}_{k}\right)$ and $\tilde{P}_{\beta, k}\left(\xi, \bar{c}_{k}\right)$, respectively, built based on the Reynolds and Favre-averaged moments, respectively, of the $c(\mathbf{x}, t)$-field. 


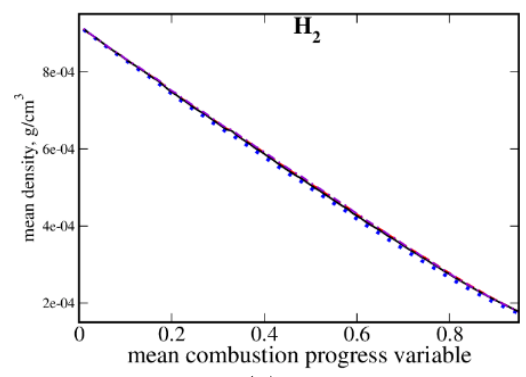

(a)

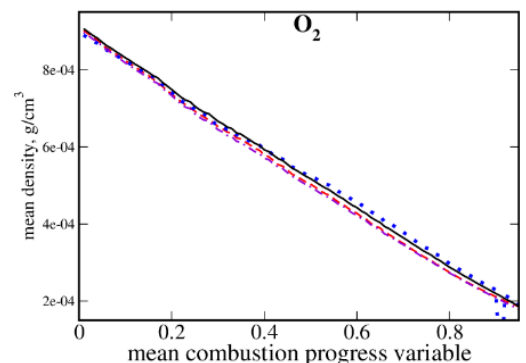

(d)

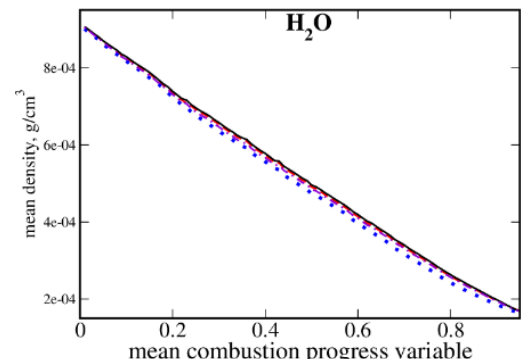

(g)

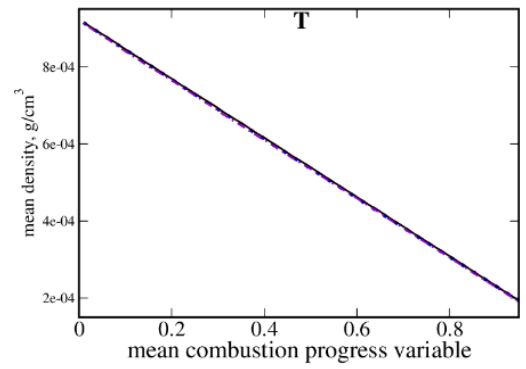

(j)

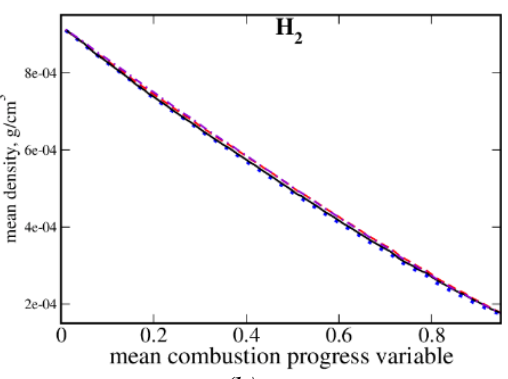

(b)

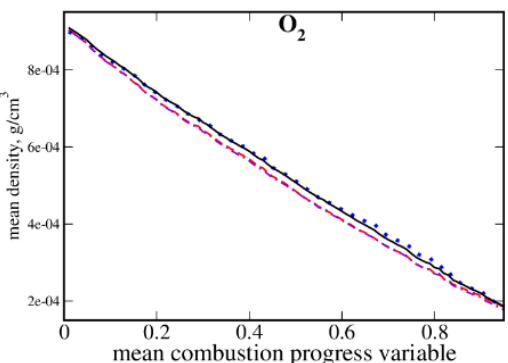

(e)

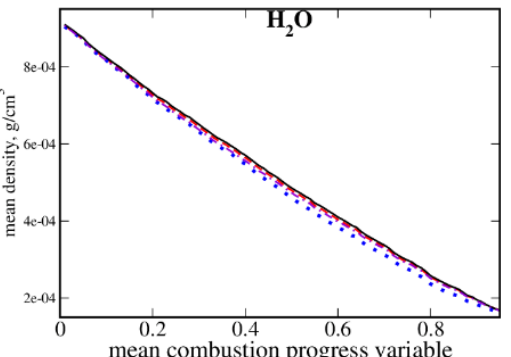

(h)

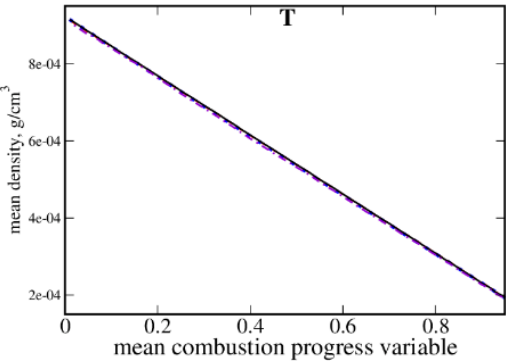

(k)

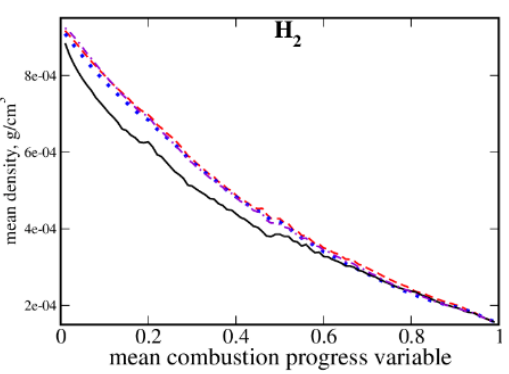

(c)

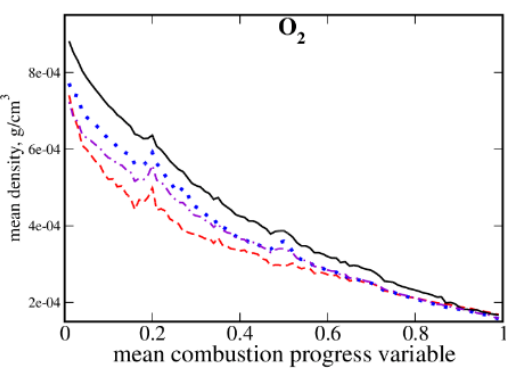

(f)

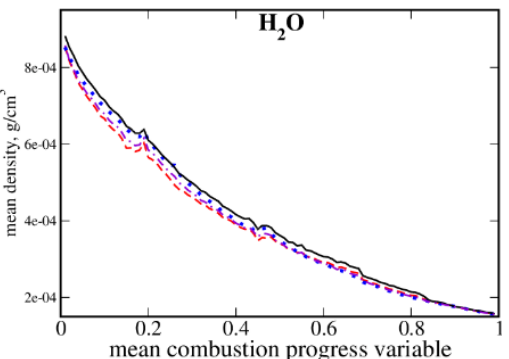

(i)

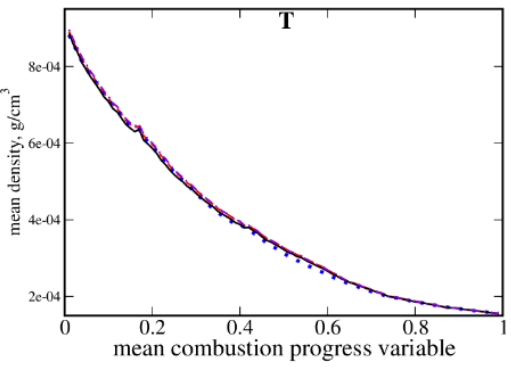

(l)

Fig. 2. Mean density $\bar{\rho}$ of the mixture vs. differently defined mean combustion progress variables $\bar{c}_{k}$. Solid lines show $\bar{\rho}\left(\bar{c}_{k}\right)$ extracted directly from the DNS data. Dashed, dotted, and dotted-dashed lines show $\bar{\rho}\left(\bar{c}_{k}\right)$ evaluated by averaging the laminarflame profiles of $\rho_{L}\left(c_{k}\right)$ and adopting the actual PDFs $P_{k}\left(\xi, \bar{c}_{k}\right)$ extracted from the DNS data and the $\beta$-function PDFs $P_{\beta, k}\left(\xi, \bar{c}_{k}\right)$ and $\widetilde{P}_{\beta, k}\left(\xi, \bar{c}_{k}\right)$, respectively. Other details are provided in the caption of Fig. 1. 


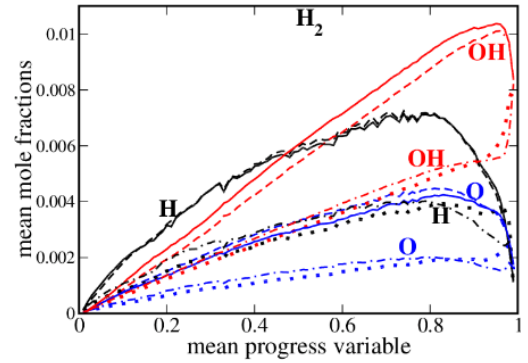

(a)

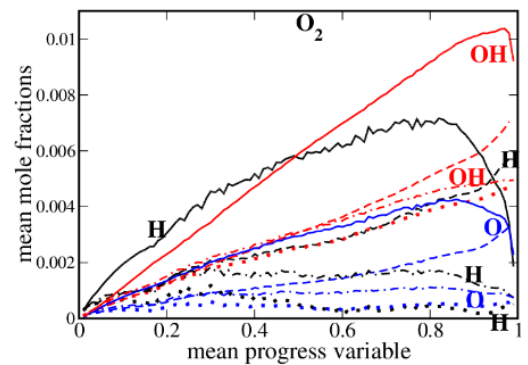

(d)

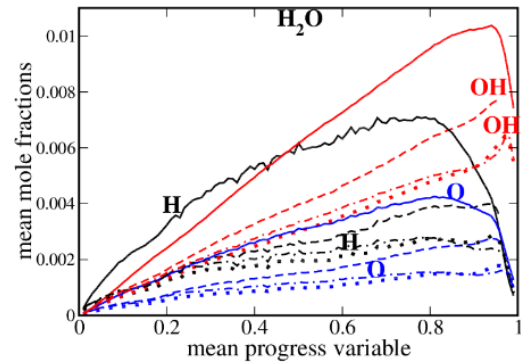

(g)

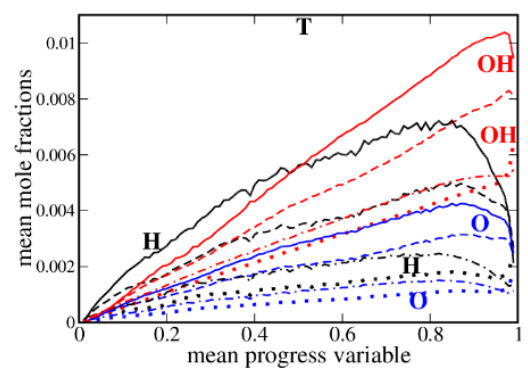

(j)

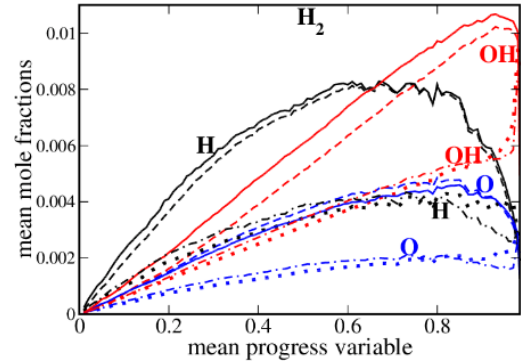

(b)

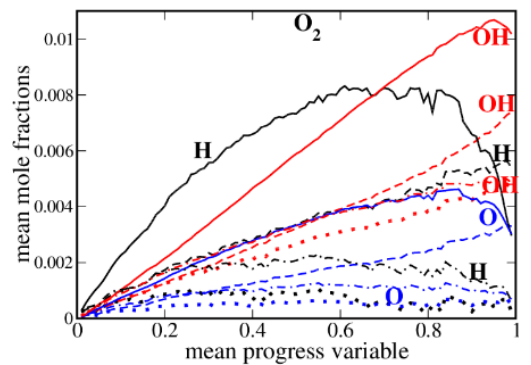

(e)

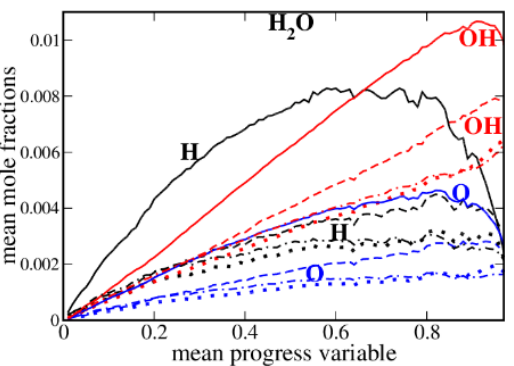

(h)

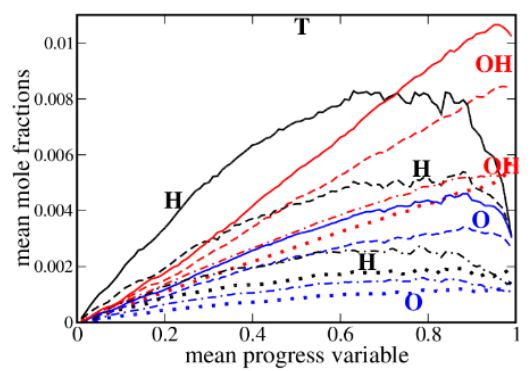

(k)

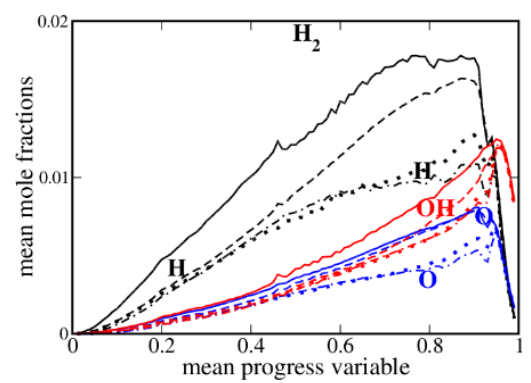

(c)

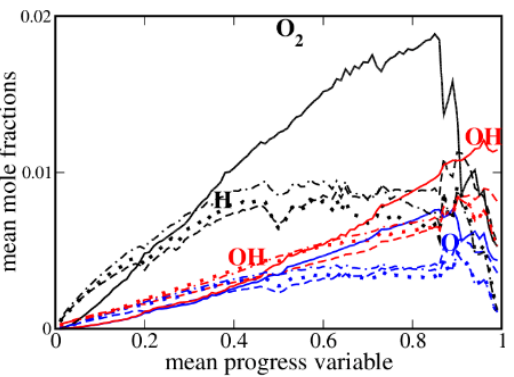

(f)

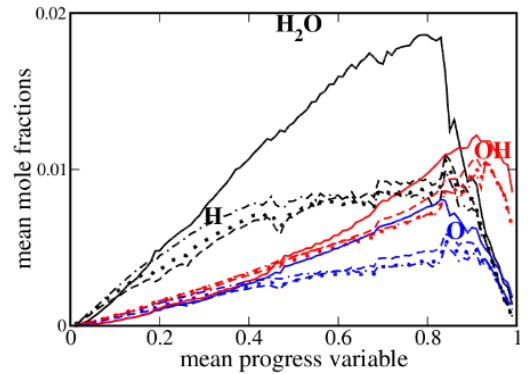

(i)

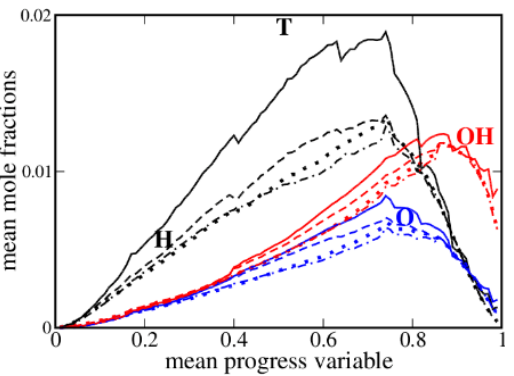

(l)

Fig. 3. Mean mole fractions $\bar{X}_{n}$ of radicals noted near relevant curves vs. differently defined mean combustion progress variables $\bar{c}_{k}$. Other details are provided in the caption of Fig. 1. 


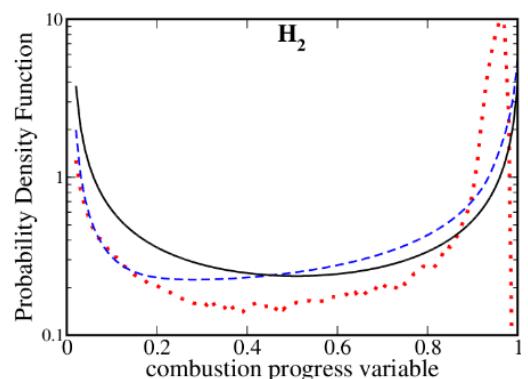

(a)

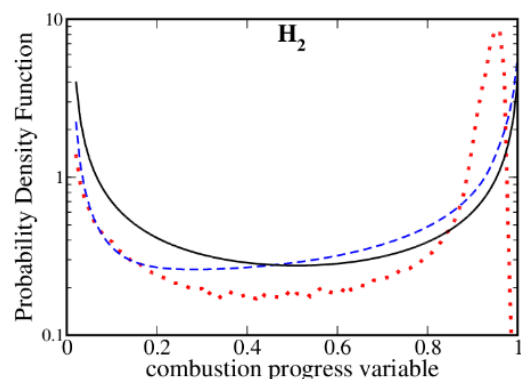

(b)

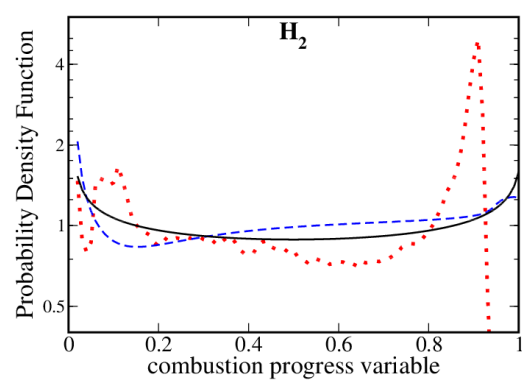

(c)

Fig. 4. PDFs $P\left(c_{F}\right)$ for the fuel-based combustion progress variable obtained at $\bar{c}_{F}=0.5$ in cases A (left panel), B (middle (panel), and C (right panel). Red dotted lines show PDFs extracted directly from the DNS data. Black solid and blue dashed lines show PDFs $P_{\beta, F}\left(\xi, \bar{c}_{F}\right)$ and $\bar{\rho}\left(\bar{c}_{F}\right) \widetilde{P}_{\beta, F}\left(\xi, \bar{c}_{F}\right) / \rho(\xi)$, respectively, obtained adopting unweighted and mass-weighted, respectively, moments of the $c(\mathbf{x}, t)$-field extracted from the DNS data. 


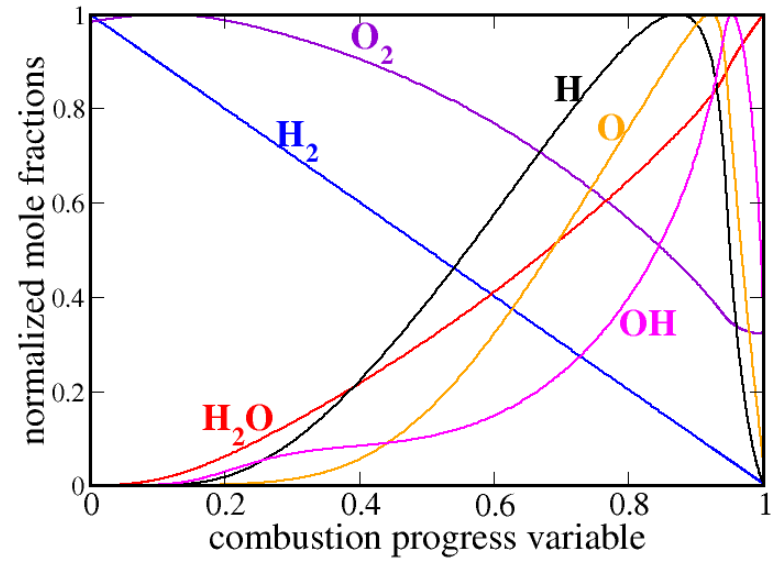

(a)

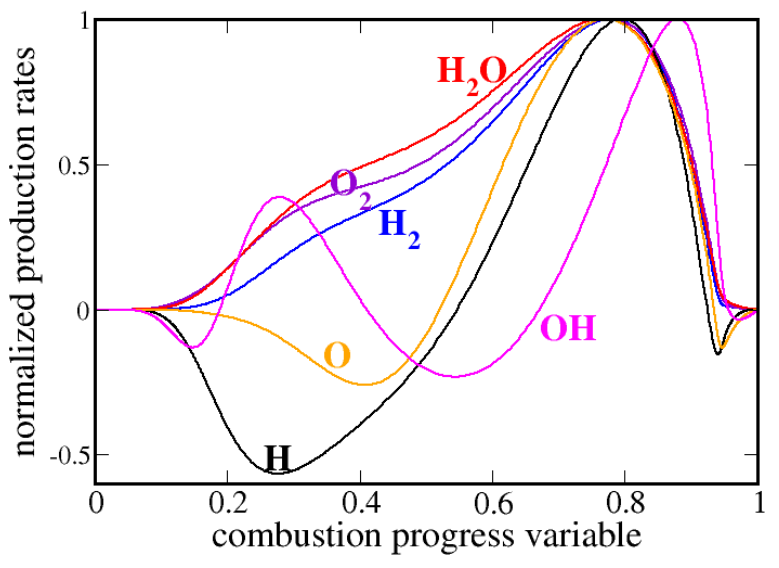

(b)

Fig. 5. Dependencies of mole fractions (left) and normalized rates (right) of production (or consumption for $\mathrm{H}_{2}$ and $\mathrm{O}_{2}$ ) of various species specified near each curve on the fuel-based combustion progress variable in the unperturbed laminar flame. 


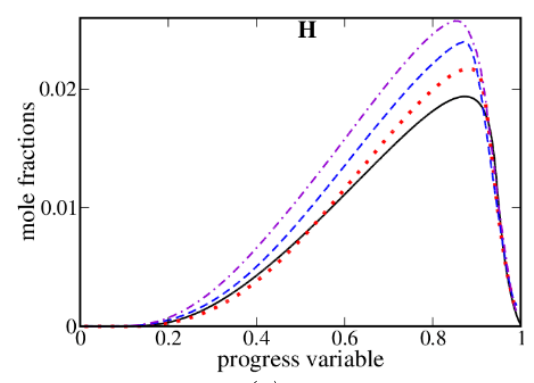

(a)

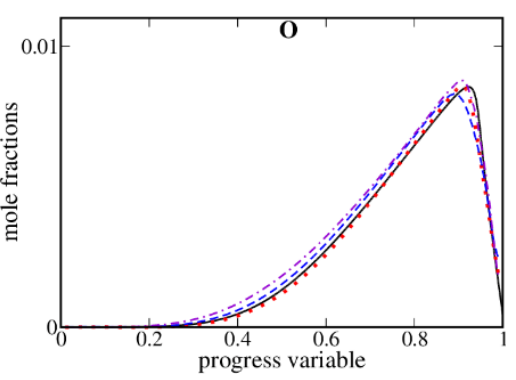

(b)

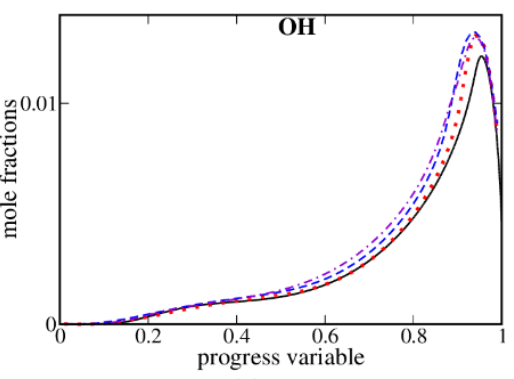

(c)

Fig. 6. Mole fractions of $\mathrm{H}$ (left panel), $\mathrm{O}$ (middle panel), and $\mathrm{OH}$ (right panel) vs. the fuel-based combustion progress variable $c_{F}$. Black solid lines show $X_{n, L}\left(c_{F}\right)$ obtained from the laminar flame. Red dotted, blue dashed, and violet dotted-dashed lines show conditioned mole fractions $\left\langle X_{n} \mid c_{F}\right\rangle$ extracted from the DNS data in cases A, B, and C, respectively. 


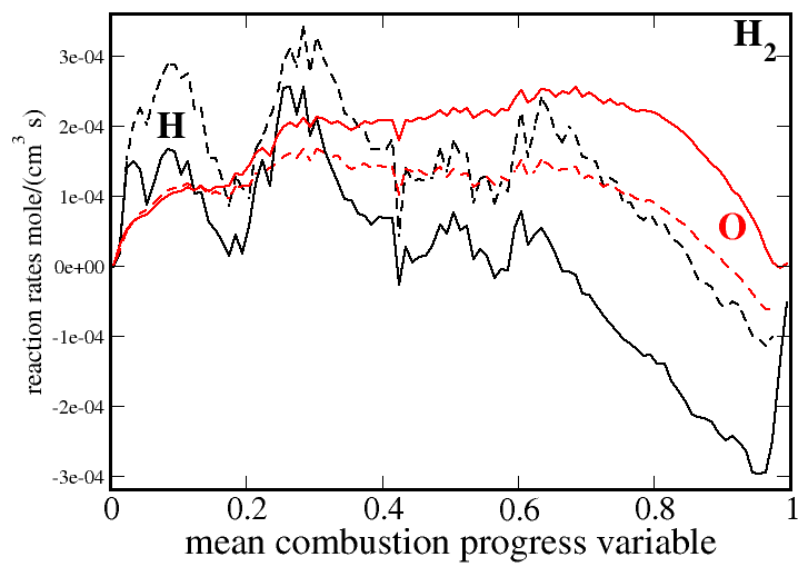

Fig. 7. Mean rates of production/consumption of radicals $\mathrm{O}$ (red curves) and $\mathrm{H}$ (black curves) vs. mean fuel-based combustion progress variable $\bar{c}_{F}$. Solid lines show $\bar{W}_{n}\left(\bar{c}_{F}\right)$ extracted directly from the DNS data. Dashed lines show $\bar{W}_{n}\left(\bar{c}_{F}\right)$ evaluated using the laminar-flame profiles $W_{n, L}\left(c_{F}\right)$ and the PDFs $P_{F}\left(\xi, \bar{c}_{F}\right)$ extracted from the DNS data. Case A. 


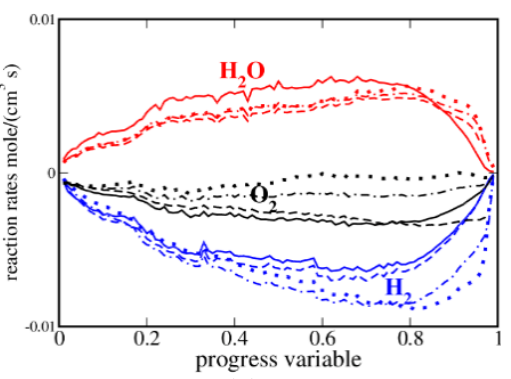

(a)

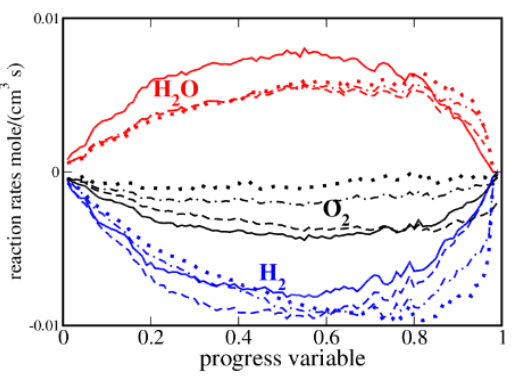

(b)

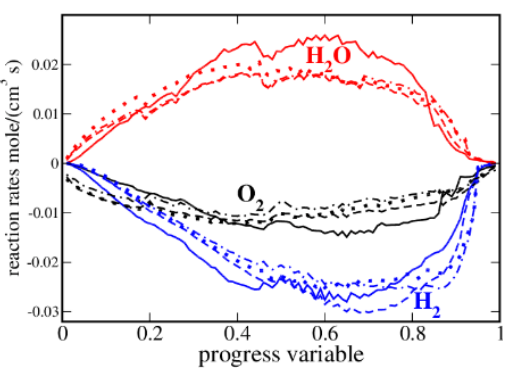

(c)

Fig. 8. Mean rates of production/consumption of various species noted near each curve vs. mean combustion progress variable defined using the mass fraction of the same species. Solid lines show $\bar{W}_{k}\left(\bar{c}_{k}\right)$ extracted directly from the DNS data. Dashed lines show $\bar{W}_{k}\left(\bar{c}_{k}\right)$ evaluated using Eq. (1), the laminar-flame profiles $W_{k, L}\left(c_{k}\right)$, and the PDFs $P_{k}\left(\xi, \bar{c}_{k}\right)$ extracted from the DNS data. Dotted and dotted-dashed lines show $\bar{W}_{k}\left(\bar{c}_{k}\right)$ calculated adopting the $\beta$-function PDFs $P_{\beta, k}\left(\xi, \bar{c}_{k}\right)$ and $\widetilde{P}_{\beta, k}\left(\xi, \bar{c}_{k}\right)$, respectively, built based on the Reynolds and Favre-averaged moments, respectively, of the $c(\mathbf{x}, t)$-field. Results obtained from flames A, B, and C are displayed in the left, middle, and right plots, respectively. 


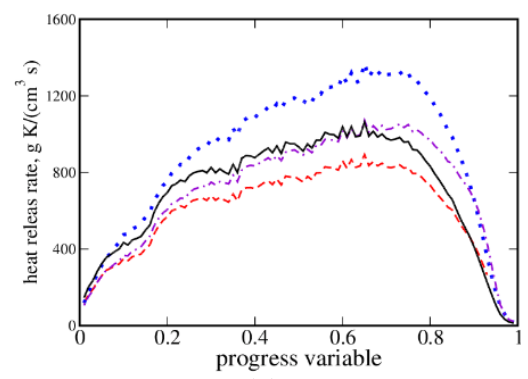

(a)

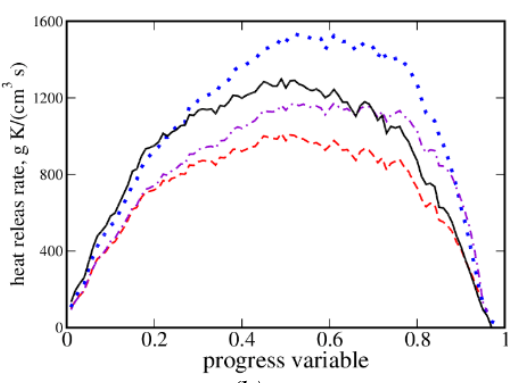

(b)

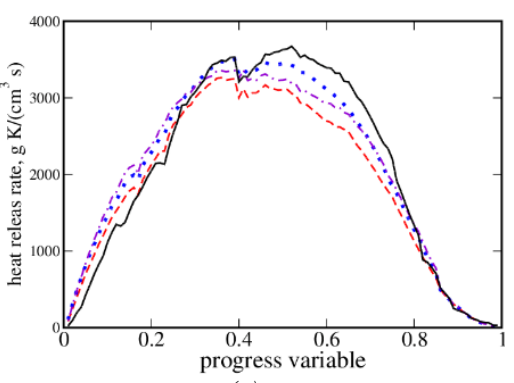

(c)

Fig. 9. Mean heat release rates $\bar{W}_{T}\left(\bar{c}_{T}\right)$ vs. mean temperature-based combustion progress variable $\bar{c}_{T}$. Legends are explained in the caption of Fig. 8. 


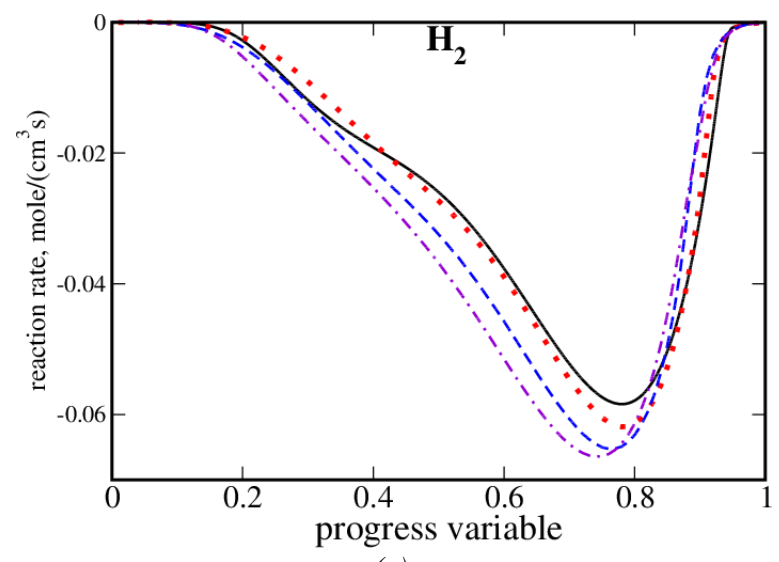

(a)

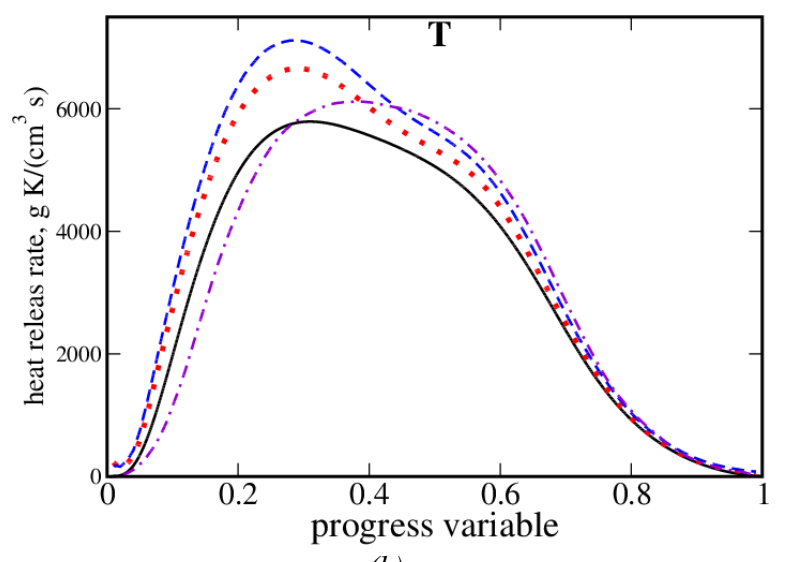

(b)

Fig. 10. Rate of fuel consumption (left panel) and heat-release rate (right panel) vs. the fuel-based and temperature-based combustion progress variables, respectively. Black solid lines show the rates obtained from the laminar flame. Red dotted, blue dashed, and violet dotted-dashed lines show conditioned rates $\left\langle W_{F} \mid c_{F}\right\rangle$ (left panel) and $\left\langle W_{T} \mid c_{T}\right\rangle$ (right panel) extracted from the DNS data in cases A, B, and C, respectively. 


\section{TABLES}

Table 1. Relevant parameters characterizing the DNS cases.

\begin{tabular}{cccccc}
\hline Case & $\boldsymbol{u}^{\prime} / \boldsymbol{S}_{\boldsymbol{L}}$ & $\boldsymbol{L}_{\boldsymbol{T}} / \boldsymbol{\delta}_{\boldsymbol{L}}$ & $\boldsymbol{R} \boldsymbol{e}_{\boldsymbol{T}}$ & $\boldsymbol{D a}$ & $\boldsymbol{K a}$ \\
\hline $\mathrm{A}$ & 0.7 & 14 & 227 & 20 & 0.75 \\
$\mathrm{~B}$ & 5 & 14 & 1623 & 2.8 & 14.4 \\
$\mathrm{C}$ & 14 & 4 & 1298 & 0.29 & 126 \\
\hline
\end{tabular}


Table 2. Time-averaged turbulent burning velocities.

\begin{tabular}{|c|c|c|c|c|}
\hline & \multirow{2}{*}{ Method } & \multicolumn{3}{|c|}{ case } \\
\hline & & A & B & $\mathrm{C}$ \\
\hline \multirow{4}{*}{$c_{F}$} & DNS & 4.56 & 7.23 & 4.52 \\
\hline & Eq. (1), actual PDF & 4.90 & 8.27 & 4.66 \\
\hline & conventional $\beta$-PDF & 7.10 & 10.6 & 4.73 \\
\hline & mass-weighted $\beta$-PDF & 5.81 & 8.42 & 4.09 \\
\hline \multirow{4}{*}{$c_{O}$} & DNS & 4.86 & 7.69 & 4.78 \\
\hline & Eq. (1), actual PDF & 6.35 & 8.91 & 5.89 \\
\hline & conventional $\beta$-PDF & 1.08 & 1.67 & 4.62 \\
\hline & mass-weighted $\beta$-PDF & 2.39 & 3.51 & 4.70 \\
\hline \multirow{4}{*}{$c_{P}$} & DNS & 4.55 & 7.19 & 4.46 \\
\hline & Eq. (1), actual PDF & 4.09 & 5.88 & 3.96 \\
\hline & conventional $\beta$-PDF & 4.87 & 7.13 & 4.24 \\
\hline & mass-weighted $\beta$-PDF & 4.34 & 6.36 & 4.00 \\
\hline \multirow{4}{*}{$c_{T}$} & DNS & 4.49 & 7.05 & 4.28 \\
\hline & Eq. (1), actual PDF & 3.93 & 5.76 & 3.96 \\
\hline & conventional $\beta$-PDF & 5.94 & 8.90 & 4.29 \\
\hline & mass-weighted $\beta$-PDF & 4.93 & 7.49 & 3.95 \\
\hline
\end{tabular}

